ARTICLE

DOI: $10.1038 / \mathrm{s} 41467-017-00551-2$

\title{
Endothelial adenosine A2a receptor-mediated glycolysis is essential for pathological retinal angiogenesis
}

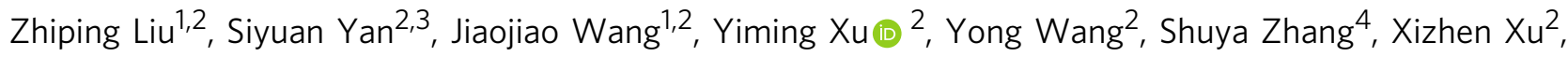
Qiuhua Yang1,2, Xianqiu Zeng1,2, Yaqi Zhou'1,2, Xuejiao Gư ${ }^{4}$, Sarah Lu², Zhongjie Fu' ${ }^{5}$ David J. Fulton ${ }^{2}$, Neal L. Weintraub ${ }^{2}$, Ruth B. Caldwell ${ }^{2}$, Wenbo Zhang ${ }^{6}$, Chaodong Wu (1) ${ }^{7}$, Xiao-Ling Liu ${ }^{4}$, Jiang-Fan Chen ${ }^{4,8}$, Aftab Ahmad ${ }^{9}$, Ismail Kaddour-Djebbar ${ }^{10}$, Mohamed Al-Shabrawey ${ }^{11}$, Qinkai Li ${ }^{1}$, Xuejun Jiang ${ }^{3}$, Ye Sun (D) ${ }^{5}$, Akrit Sodhi ${ }^{12}$, Lois Smith ${ }^{5}$, Mei Hong ${ }^{1} \&$ Yuqing Huo ${ }^{2}$

Adenosine/adenosine receptor-mediated signaling has been implicated in the development of various ischemic diseases, including ischemic retinopathies. Here, we show that the adenosine A2a receptor (ADORA2A) promotes hypoxia-inducible transcription factor-1 (HIF-1)-dependent endothelial cell glycolysis, which is crucial for pathological angiogenesis in proliferative retinopathies. Adora2a expression is markedly increased in the retina of mice with oxygen-induced retinopathy (OIR). Endothelial cell-specific, but not macrophagespecific Adora2a deletion decreases key glycolytic enzymes and reduces pathological neovascularization in the OIR mice. In human primary retinal microvascular endothelial cells, hypoxia induces the expression of ADORA2A by activating HIF-2 $\alpha$. ADORA2A knockdown decreases hypoxia-induced glycolytic enzyme expression, glycolytic flux, and endothelial cell proliferation, sprouting and tubule formation. Mechanistically, ADORA2A activation promotes the transcriptional induction of glycolytic enzymes via ERK- and Akt-dependent translational activation of HIF-1 $\alpha$ protein. Taken together, these findings advance translation of ADORA2A as a therapeutic target in the treatment of proliferative retinopathies and other diseases dependent on pathological angiogenesis.

\footnotetext{
${ }^{1}$ Drug Discovery Center, Key Laboratory of Chemical Genomics, Peking University Shenzhen Graduate School, Shenzhen 518055, China. ${ }^{2}$ Vascular Biology Center, Department of Cellular Biology and Anatomy, Medical College of Georgia, Augusta University, Augusta, GA 30912, USA. ${ }^{3}$ State Key Laboratory of Mycology, Institute of Microbiology, Chinese Academy of Science, Beijing 100101, China. ${ }^{4}$ Molecular Neuropharmacology Laboratory, School of Optometry and Ophthalmology and Eye Hospital, Wenzhou Medical University, Wenzhou 325035, China. ${ }^{5}$ Department of Ophthalmology, Boston Children's Hospital, Harvard Medical School, Boston, MA 02115, USA. ${ }^{6}$ Department of Ophthalmology \& Visual Sciences, University of Texas Medical Branch (UTMB), Galveston, TX 77555, USA. ${ }^{7}$ Department of Nutrition and Food Science, Texas A\&M University, College Station, TX 77843, USA. ${ }^{8}$ Department of Neurology, Boston University School of Medicine, Boston, MA 02118, USA. ${ }^{9}$ Department of Anesthesiology and Perioperative Medicine, University of Alabama at Birmingham, Birmingham, AL 35249, USA. ${ }^{10}$ Department of Physiology, Medical College of Georgia, Augusta University, Augusta, GA 30912, USA. ${ }^{11}$ James and Jean Culver Vision Discovery Institute, Medical College of Georgia, Augusta University, Augusta, GA 30912, USA. ${ }^{12}$ Wilmer Eye Institute, Johns Hopkins School of Medicine, Baltimore, MD 21287, USA. Correspondence and requests for materials should be addressed to

M.H. (email: meihong@pkusz.edu.cn) or to Y.H. (email: yhuo@augusta.edu)
} 
$\mathrm{P}$ athological angiogenesis is among the most common causes of irreversible blindness for individuals at all ages, including newborns (retinopathy of prematurity), middleage adults (proliferative diabetic retinopathy, PDR) and the elderly (age-related macular degeneration, AMD). Retinal neovascularization is one of the major pathologies for these sightthreatening retinopathies ${ }^{1-3}$. Neovascular tissues are characterized by incompetent, leaky blood vessels that can bleed or contract, leading to hemorrhage or retinal detachment and eventually to blindness ${ }^{1}$. Increased endothelial sprouting and proliferation are major cellular events causing pathological proliferative retinopathies ${ }^{4} 5$. Therefore, deciphering the molecular mechanisms underlying these early cellular events is key to understanding and further developing novel therapeutic approaches for the prevention or treatment of these visionthreatening diseases.

Increased emerging evidence indicates that not only signals from growth factors and the Notch pathway, but also glucose metabolism, control endothelial cell (EC) proliferation, migration, and neovascularization 6,7 . ECs rely on glycolysis rather than oxidative metabolism for ATP production and vessel sprouting ${ }^{8}$. Reduction of glycolysis using an inhibitor of 6-phosphofructo-2kinase/fructose-2, 6-bisphosphatase isoform 3 (PFKFB3) or endothelial-specific genetic deletion of $P f k f b 3$ inhibits pathological angiogenesis in murine models of AMD and oxygen-induced retinopathy (OIR), respectively ${ }^{9}{ }^{10}$. Importantly, increased glycolysis, evidenced by an increased level of lactate in vitreous fluid, has been demonstrated in patients with $\mathrm{PDR}^{11}$. Due to this close association between EC glycolysis and pathological retinal angiogenesis as well as substantial demand for new treatment of retinopathies, it is pressing to uncover practical targeting molecules that regulate the glycolytic pathway in retinal ECs.

Hyperactivation of adenosine signaling has been implicated in cellular responses to hypoxia and the development of various ischemic diseases ${ }^{12}$. Loss of functional vasculature and consequent hypoxia precedes the development of ischemic proliferative retinopathies. Hypoxia results in marked increases in adenosine production and adenosine receptor signaling ${ }^{12}$. Indeed, in a canine model of OIR, peak adenosine levels in the retina correlated temporally with active vasculogenesis in the retina ${ }^{13}$. Immunoreactivity of adenosine A2a receptor (Adora2a), one of the adenosine receptors, is prominent in ECs and angioblasts in newly formed blood vessels, and is significantly elevated in intravitreal neovascularization ${ }^{14}$. Yet it remains unclear whether retinal endothelial adenosine-Adora2a signaling plays a role in glycolysis and pathological retinal angiogenesis, although in mouse models of wound healing and hind limb ischemia, activation of Adora2a brings about beneficial angiogenesis ${ }^{15}, 16$.

In this study, we showed that Adora2a expression is significantly increased in pathological retinal neovessels in OIR. We found that hypoxia upregulates ADORA2A expression by activating hypoxia-inducible transcription factor (HIF) $-2 \alpha$ in human microvascular retinal ECs (HRMECs). Using gain- and loss-of-function approaches, we identified ADORA2A as a key regulator of the metabolic and angiogenic switch in HRMECs in vitro. Our study further demonstrated that endotheliumspecific Adora2a deletion reduces glycolysis and pathological neovascularization in retinopathy in vivo.

\section{Results}

Expression of Adora2a in retinal pathological angiogenesis. To study the role of adenosine receptors (ADORs) in pathological angiogenesis, we first assessed the expression profile of ADORs in the retinas of a mouse OIR model (Fig. 1a). Real-time PCR analysis revealed that expression of the Adora2a gene was significantly increased while adenosine $\mathrm{A}_{1}$ receptor (Adora1) and adenosine $A_{2 B}$ receptor (Adora $2 b$ ) levels were reduced on postnatal day (P)17 in OIR retinas, compared with controls in room air (RA) (Fig. 1b). We next performed a time-course analysis of messenger RNA (mRNA) levels of Adors from P7 to P12 (the hyperoxia phase), and P12 to p17 (the hypoxic-ischemic phase) of OIR retinas. We found no noticeable changes in the expression of Adors from P7 to P12 (Fig. 1c), whereas expression of Adora $2 a$ steadily increased from P12 to P17 (Fig. 1d), indicative of a sustained increase in the expression of Adora2a throughout the hypoxic-ischemic phase of OIR. To localize the expression of Adora2a, we performed doubleimmunofluorescence staining of whole-mount retinas from OIR or control mice using a well-characterized monoclonal antibody for Adora $2 \mathrm{a}^{17,18}$, and a retinal blood vessel marker (Isolectin B4), or a macrophage/microglia marker (IBa1). In RA control retinas, Adora2a was present in the blood vessel wall, whereas in OIR retinas, Adora2a was strongly expressed within and around pathological neovascular tufts, particularly in and around ECs and macrophages/microglias, as indicated by its colocalization with blood vessels and IBa1 (Fig. 1e, f). Ablation of Adora2a expression in retinas of global homozygous Adora2a knockout mice (Adora $2 a^{-/-}$, Supplementary Fig. 1) confirmed antibody specificity. Using laser-capture microdissection to isolate pathological neovascular tufts from OIR mice and normal vessels from control (RA) mice, we confirmed that Adora2a mRNA level is higher in retinal neovessels compared with normal vessels (Fig. 1g). Importantly, type 1 diabetic patients homozygous for the T allele of ADORA2A SNP rs2236624 and rs4822489, two genotypes associated with low incidence of $\mathrm{PDR}^{19}$, had lower levels of ADORA2A mRNA compared with their controls, which are type 1 diabetic patients homozygous for the $\mathrm{C}$ allele of ADORA2A SNP rs2236624 and G allele of rs4822489 (Supplementary Fig. 2). These findings indicate a close association of a high level of ADORA2A expression with proliferative retinopathies.

Retinal neovascularization in mice lacking endothelial Adora2a. To investigate the biological significance of Adora2a expressed on vascular ECs or macrophages in retinal neovascularization, Cdh5-Cre and Lysm-Cre mice were bred with Adora2 $a^{\text {flox/flox }}$ mice to generate Adora2 $a^{\text {flox/flox }}$; Cdh5-Cre

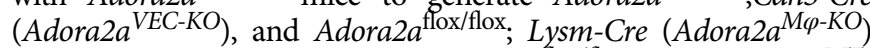
mice, respectively. The littermate Adora $2 a^{\text {flox/flox }}$ mice $\left(\right.$ Adora $2 a^{W T}$ ) mice were used as wild-type (WT) controls. After mice were subjected to the OIR, we performed isolectin B4 analysis of wholemount retinas and observed that both EC- and macrophage-specific Adora $2 a$ deletion reduced the avascular area compared with the avascular area in Adora $2 a^{W T}$ mice at P17 in the OIR model (Fig. 2a). However, with the loss of endothelial, but not macrophage Adora $2 a$, we observed significantly less pathological neovascularization compared with WT controls $(7.85 \pm 0.33 \%$ vs. $3.31 \pm 0.39 \%$ of total retina area; $P<0.001$ using one-way ANOVA followed by Bonferroni test; Fig. 2a). To assess whether increasing the ischemic drive could reveal a role for macrophage Adora $2 a$ on retinal neovascularization, we exposed mice to $75 \%$ (rather than $70 \%$ ) $\mathrm{O}_{2}$; this resulted in a larger area of avascular retina, but failed to demonstrate a decrease in pathological neovascularization with loss of macrophage-specific Adora2a compared to WT controls (Fig. 2b). Collectively, these results suggest that genetic Adora2a deletion from ECs but not macrophages suppresses pathological neovascularization during OIR. We also investigated the effect of endothelialspecific Adora2a deletion on vascular regression at P12 and observed that there was no significant difference in retinal vaso-obliteration following the hyperoxic phase between the Adora2a $a^{V E C-K O}$ and 
a

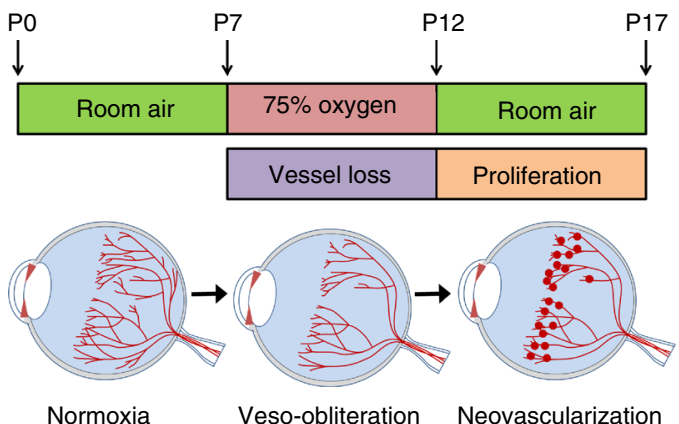

b

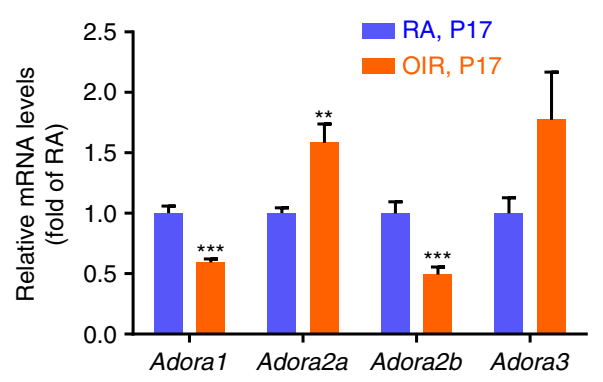

C

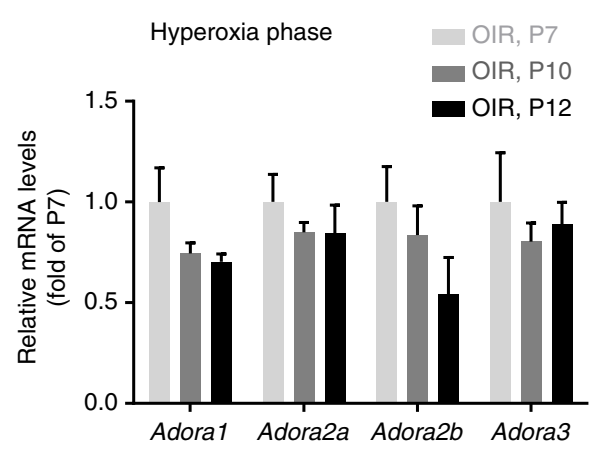

d

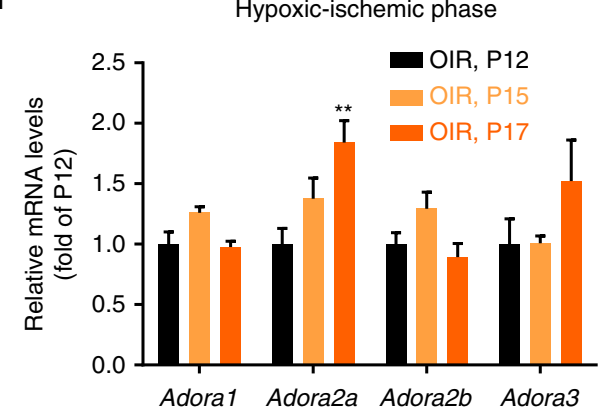

e

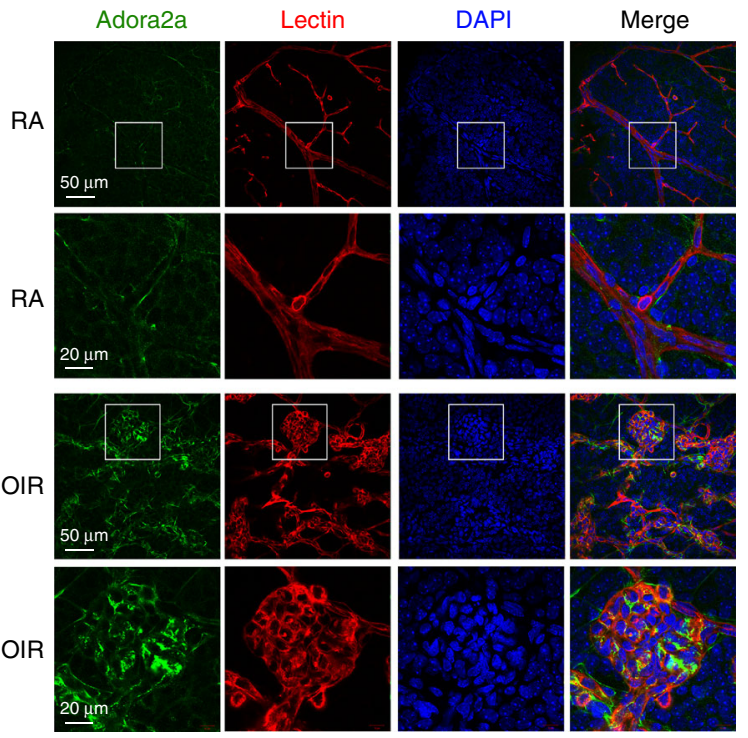

f

Adora2a

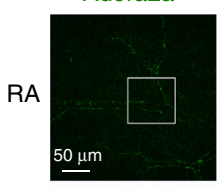

IBa1

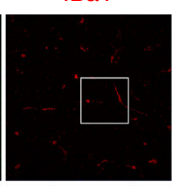

DAPI

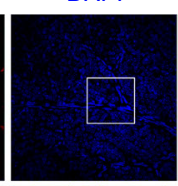

Merge

RA
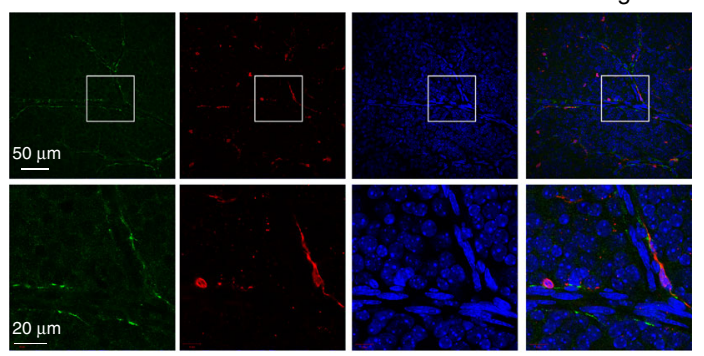

OIR
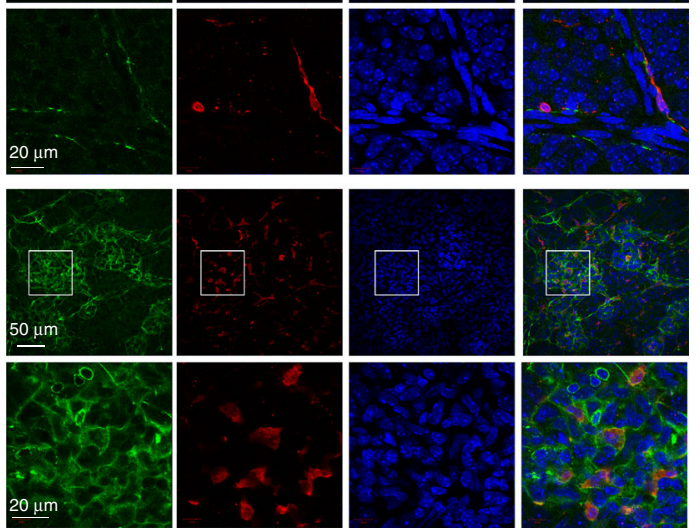

g
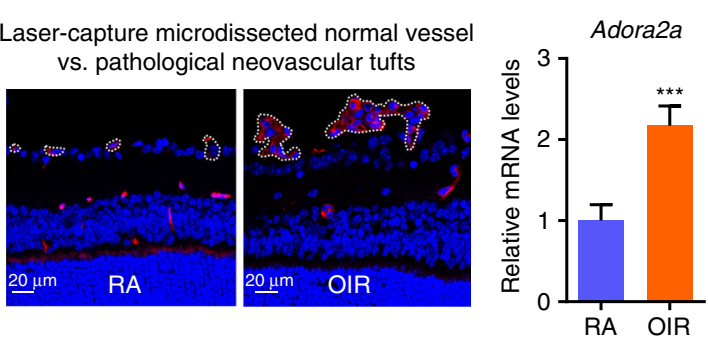

Fig. 1 Localization and expression of Adora2a in rodent proliferative retinopathy. a Schematic illustration of mouse OIR model. Neonatal mice with nursing mothers were exposed to $75 \% \mathrm{O}_{2}$ from postnatal day (P) 7 to $\mathrm{P} 12$, followed by room air $(R A)$ with maximum neovascularization at P17. b Real-Time PCR analysis of Adora1, Adora2a, Adora2b, and Adora3 mRNA expression in the whole retina. Retinas were from RA or OIR mice at P17. ${ }^{\star \star \star} P<0.001$ vs. RA group ( $n=7$ mice per group). c, d Real-Time PCR analysis of adenosine receptor mRNA expression in the whole retina. Retinas were obtained from mice at the times indicated. Data were normalized to both the expression of internal control and to gene mRNA expression of each RA control at each time point. ${ }^{\star \star} P$ $<0.01$ vs. P12 ( $n=4$ mice per group for $\mathbf{c}$ and $n=7$ mice per group for $\mathbf{d}$ ). e, $\mathbf{f}$ Localization and expression of Adora2a in the RA and OIR retinas.

Retinopathy was induced in wild-type mice. P17 RA and OIR retinas were stained with Adora2a (green), isolectin B4 (Lectin, red, vessel, e), or IBa1 (red, macrophages/microglias, f) and DAPI (blue, nuclei). In all, 2nd and 4th rows are magnification of the boxed regions in the 1st and 3rd rows, respectively. Scale bar: $50 \mu \mathrm{m}$ (1st and 3rd rows) and $20 \mu \mathrm{m}$ (2nd and 4th rows). g Real-Time PCR analysis of Adora2a mRNA expression in laser-capture microdissected pathological neovessels (tufts) from OIR mice compared with normal vessels from control mice raised in RA at P17. ${ }^{\star \star \star} P<0.001$ vs. RA ( $n=4$ per group). Data are represented as means \pm s.e.m. Statistical significance was determined by unpaired Student's $t$-test (for $\mathbf{b}, \mathbf{g}$ ) and one-way ANOVA followed by Bonferroni test (for $\mathbf{c}, \mathbf{d}$ ) 
Adora2a $a^{W T}$ groups (Supplementary Fig. 3), indicating that the reduced avascular area in Adora $2 a^{V E C-K O}$ mice at P17 is ascribed to the modulation of revascularization at the hypoxic phase, rather than the alteration of vaso-obliteration at the hyperoxic phase.

To study whether endothelial-specific deletion of Adora2a affected developmental angiogenesis in the retina, we analyzed the development of the retinal vascular networks at P5, P7, P12, and P17 of Adora $2 a^{V E C-K O}$ and control mice under RA by isolectin B4 staining of whole-mounted retinas. Quantitative analysis of the vascularized retinal area at P5 and P7, as well as the vascular density of three retinal vascular layers (the superficial, intermediate and deep layers) at P12 and P17, found no significant difference in the distribution and density of retinal vascularization between Adora $2 a^{V E C-K O}$ and Adora $2 a^{W T}$ mice (Supplementary Fig. 4).
To further evaluate hypoxia-induced pathologic angiogenesis in the retinas of these animals, we performed hematoxylin and eosin (H\&E) and Ki-67 staining. Histologic examination revealed that OIR-induced infiltration of non-ganglion cells and neovascular nuclei from the inner limiting membrane into the vitreous were significantly reduced in Adora $2 a^{V E C-K O}$ mice, compared with that of WT mice (Fig. 2c, d). Additionally, we observed a dramatic decrease of proliferative vascular ECs in Adora $2 a^{V E C-K O}$ mice, as assessed by double immunofluorescence staining of the proliferation marker Ki-67 and the endothelial nuclear marker ETS related gene $(E R G)^{20,} 21$ (Fig. 2e, f). Taken together, these data suggest that endothelial Adora2a activation plays a causal role in pathological angiogenesis in the OIR model. a
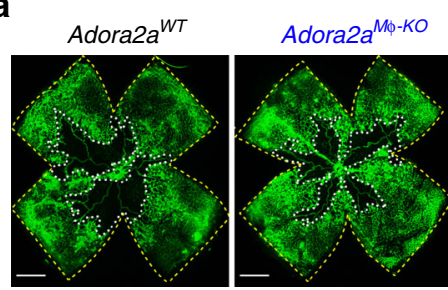

b
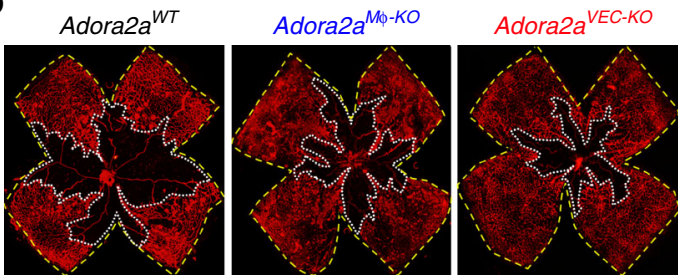

Adora2a ${ }^{\text {VEC-KO }}$

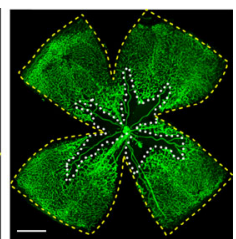

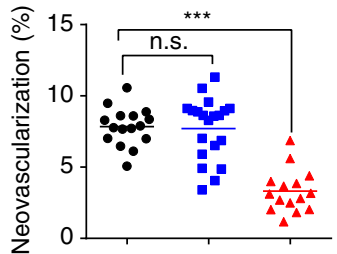

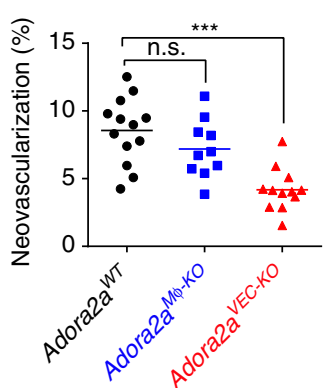

e
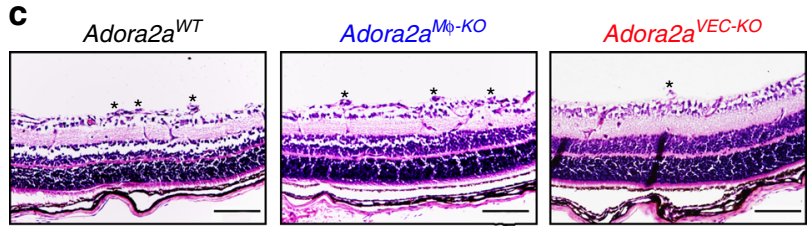

d

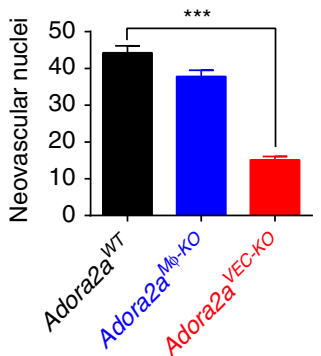

f

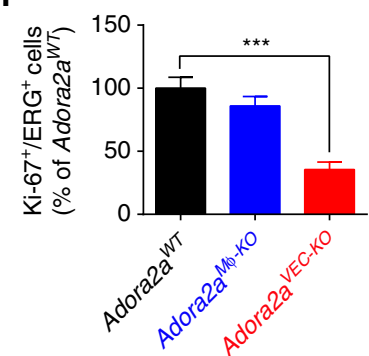

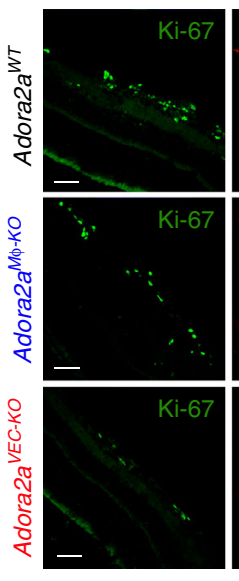
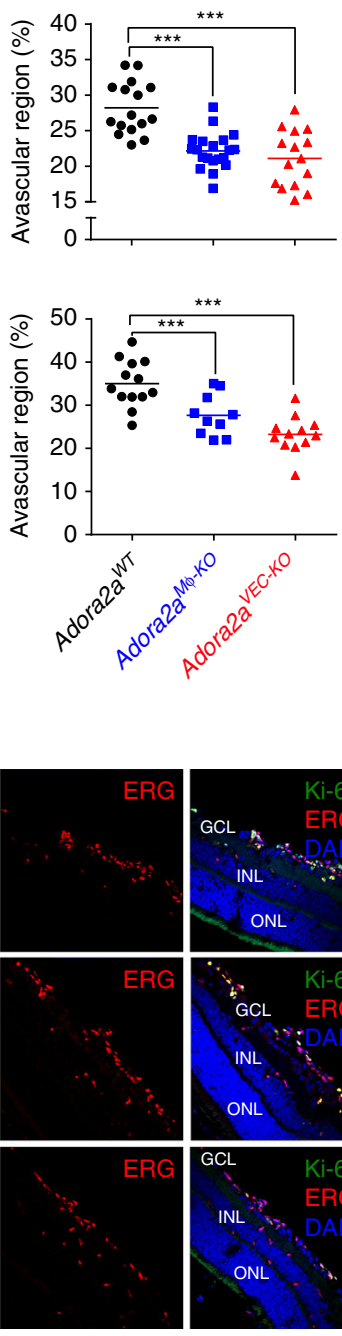

Fig. 2 Endothelial Adora2a deficiency significantly decreases formation of pathological neovascularization in OIR retinas. a, b Quantification of pathological neovascularization and vessel dropout area (within the white borders) in postnatal day (P) 17 OIR retinas. Adora2 $a^{\text {flox/flox }}(A d o r a 2 a W T$ ), Adora2 $a^{\text {flox/flox }}$ Lysm ${ }^{\text {cre/cre }}\left(A d o r a 2 a^{M \varphi-K O}\right.$ ), and Adora2 $a^{\text {flox } / \text { flox }} C$ dh5 ${ }^{\text {cre }}$ (Adora2aVEC-KO) mice were exposed to $70 \% \mathrm{O}_{2}$ a or $75 \% \mathrm{O}_{2} \mathbf{b}$. Areas of

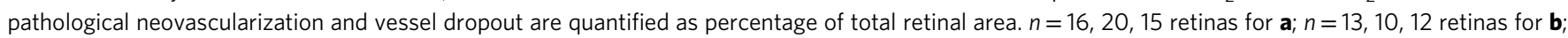
${ }^{\star \star \star} P<0.001$ vs. Adora2aWT group. Scale bars: $1000 \mu \mathrm{m}$. c Histological analysis of infiltration of neovascular nuclei from inner limiting membrane into vitreous in the OIR retinas. Nuclei on the vitreal side of the inner limiting membrane are indicated by asterisk. Scale bars: $100 \mu \mathrm{m}$. $\mathbf{d}$ Quantitative analysis of the number of neovascular nuclei in the OIR retinas. ${ }^{\star} P<0.001$ ( $n=6$ mice for Adora2aWT group; $n=8$ for $A d o r a 2 a^{M} \varphi-K O$ and Adora2 ${ }^{V E C-K O}$ groups). e Ki-67 immunofluorescent staining on OIR retinas. Representative green (Ki-67), red (ERG), blue (nuclei, DAPI), and merged images were captured with confocal fluorescent microscopy. GCL ganglion cell layer, INL inner nuclear layer, ONL outer nuclear layer. Scale bars: 50 mm. f Quantitative analysis of the Ki-67 and ERG double-positive cells in each group. ${ }^{\star} P<0.001$ ( $n=9$ mice for each group). Data are represented as means \pm s.e.m. Statistical significance was determined by one-way ANOVA followed by Bonferroni test 
a

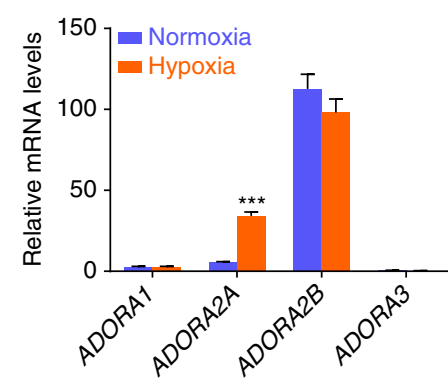

b

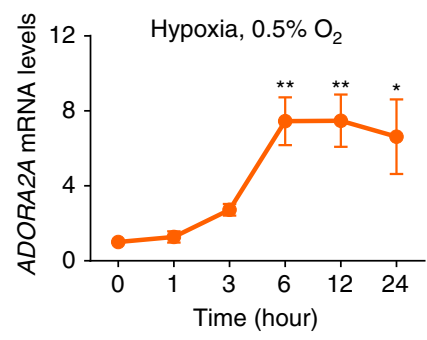

C

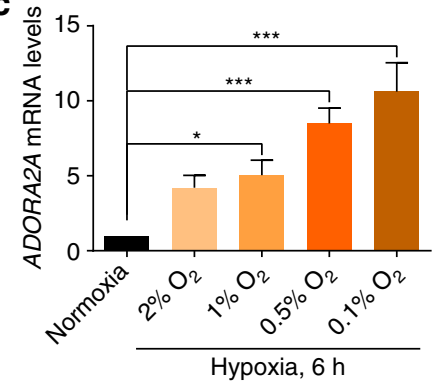

d

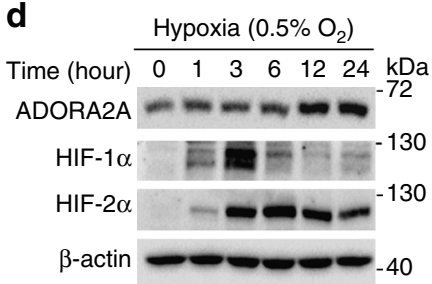

e

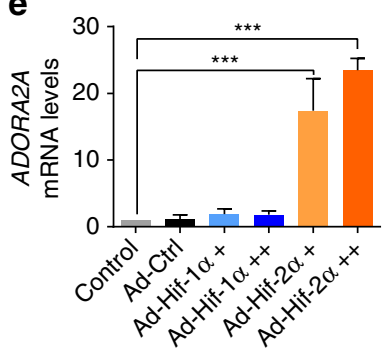

$\mathbf{f}$

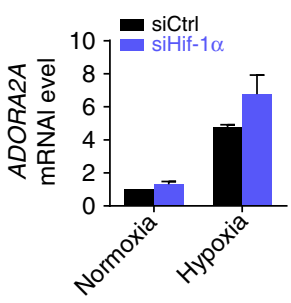

g

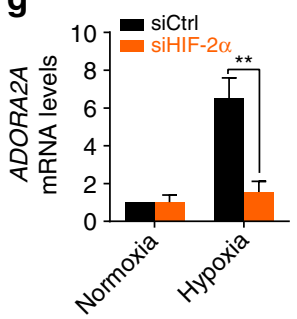

Fig. 3 Hypoxia upregulates ADORA2A expression by activating HIF-2 $\alpha$ in HRMECs. a Real-Time PCR analysis of mRNA expression for adenosine receptors in HRMECs. HRMECs were exposed to normoxia $\left(21 \% \mathrm{O}_{2}\right)$ or hypoxia $\left(0.5 \% \mathrm{O}_{2}\right)$ for 6 h. $n=4$. ${ }^{\star \star \star} P<0.001$ vs. normoxia. b, c Real-Time PCR analysis of ADORA2A mRNA expression in HRMECs. HRMECs were exposed to normoxia $\left(21 \% \mathrm{O}_{2}\right)$ or hypoxia $\left(0.5 \% \mathrm{O}_{2}\right)$ for indicated times $\mathbf{b}$ or with indicated $\mathrm{O}_{2}$ concentrations for 6 h $\mathbf{c} . n=4$. ${ }^{\star} P<0.05 ;{ }^{\star \star} P<0.01$, ${ }^{\star \star \star} P<0.001$ vs. normoxia. d Western blot analysis of ADORA2A protein expression in HRMECs exposed to hypoxia $\left(0.5 \% \mathrm{O}_{2}\right)$ for indicated times. $n=4$. HIF- $1 \alpha$ and HIF- $2 \alpha$ were used as positive controls for hypoxia, and $\beta$-actin was used as loading control. e Real-Time PCR analysis of ADORA2A mRNA expression in HRMECs. HRMECs were infected with $10(+)$ or $30(++)$ pfu per cell of either Ad-mutHIF- $1 \alpha$, Ad-mutHIF- $2 \alpha$, or Ad-Ctrl. $n=3$. ${ }^{\star} P<0.001$. $\mathbf{f}, \mathbf{g}$ Real-Time PCR analysis of ADORA2A mRNA expression in HRMECs. HRMECs were transfected with siHIF- $1 \alpha \mathbf{f}$, siHIF- $2 \alpha \mathbf{g}$, or siCtrl. Forty-eight hours later, cells were exposed to hypoxia $\left(0.5 \% \mathrm{O}_{2}\right)$ or air $\left(21 \% \mathrm{O}_{2}\right)$ for an additional 12 h. $n=4$. ${ }^{\star \star} P<0.01$. Data are represented as means \pm s.e.m. Statistical significance was determined by unpaired Student's $t$-test

Increased ADORA2A expression in hypoxic HRMECs via HIF$2 \boldsymbol{\alpha}$. Given the observation that endothelial Adora2a activation is critical for hypoxia-induced pathological angiogenesis during OIR and that Adora2a expression is increased in mouse OIR retinas, we next tested whether hypoxia upregulates ADORA2A expression in HRMECs. Strikingly, primary cultured HRMECs exposed to hypoxia exhibited a significant increase in mRNA expression of $A D O R A 2 A$, but not $A D O R A 1, A D O R A 2 B$, and $A D O R A 3$ (Fig. 3a), and the extent of this increase closely correlates with the length of exposure time and the severity of hypoxia (Fig. 3b, c). Similar changes also occurred in the expression of ADORA2A at the protein level (Fig. 3d). HIFs, particularly HIF$1 \alpha$ and HIF- $2 \alpha$, mediate expression of numerous genes under hypoxic conditions. We investigated the role of these two HIFs in regulating ADORA2A in HRMECs. To this end, we first carried out HIF gain-of-function studies using adenoviral vectors encoding mutant HIF-1 $\alpha$ (Ad-mutHIF-1 $\alpha$ ) or mutant HIF- $2 \alpha$ (Ad-mutHIF-2 $\alpha$ ), which are both stable and transcriptionally active under normoxic conditions. Elevation of HIF mRNA levels in Ad-mutHIF-infected cells was confirmed by Real-Time PCR (Supplementary Fig. 5a, b). We found that HIF-2 $\alpha$, but not HIF- $1 \alpha$, upregulated ADORA2A mRNA in HRMECs (Fig. 3e). To confirm these findings, we also performed HIF loss-of-function studies in which we silenced the gene of HIF- $1 \alpha$ or HIF- $2 \alpha$ in HRMECs using short interfering RNA (siRNA) (Supplementary Fig. 5c, d). Knockdown of HIF-2 $\alpha$, but not HIF-1 $\alpha$, reversed ADORA2A upregulation in HRMECs exposed to hypoxia (Fig. 3f, g). These findings indicate that hypoxia induces ADORA2A expression via HIF-2 $\alpha$-dependent mechanisms in HRMECs.

ADORA2A-regulated proliferation and sprouting of HRMECs. To evaluate the effect of ADORA2A on HRMEC proliferation, we performed BrdU incorporation, Ki-67 reactivity, and WST-1 proliferation assays, as well as cell number counting using HRMECs transfected with ADORA2A siRNA. Reduction of mRNA and protein levels of ADORA2A in silenced cells were confirmed by Real-Time PCR (Supplementary Fig. 6a) and western blot (Supplementary Fig. 6b), respectively. These assays consistently showed that ADORA2A knockdown markedly decreased HRMEC proliferation under hypoxia conditions (Fig. $4 \mathrm{a}-\mathrm{d}$ ). Similar results were obtained using the ADORA2A antagonist ZM241385 (Supplementary Fig. 7a, b). We found that ADORA2A knockdown also reduced EC proliferation under normoxic conditions (Supplementary Fig. 7c, d), though only modestly. Conversely, overexpression of ADORA2A by infection with adenovirus carrying the $A D O R A 2 A$ gene $\left(\mathrm{Ad}-\mathrm{A}_{2 \mathrm{~A}} \mathrm{R}\right)$ elevated ADORA2A protein levels (Supplementary Fig. 6c) and promoted HRMEC proliferation (Supplementary Fig. 7e-h). This proliferation of ADORA2A-overexpressing HRMECs was further enhanced by adding exogenous adenosine (Fig. $4 \mathrm{e}-\mathrm{i}$ ). In the threedimensional spheroid sprouting assay, ADORA2A knockdown reduced hypoxia and VEGF-induced vessel sprouting (Fig. $4 \mathrm{j}-1$ ). $A D O R A 2 A$ overexpression modestly increased sprout numbers and length under basal conditions (Supplementary Fig. 7i-k). This increased sprouting was further augmented in the presence of adenosine (Fig. 4m-o). These findings were confirmed in another angiogenesis assay, where HRMECs form a $2 \mathrm{D}$ vessel network (Fig. 4p-u). Collectively, these data indicate a significant proangiogenic effect of ADORA2A activation in HRMECs.

ADORA2A-associated glycolysis in retinal ECs. EC glycolysis plays a critical role in vessel sprouting and angiogenesis ${ }^{22}$. In response to hypoxia, HRMECs upregulated mRNA levels of key glycolytic enzymes, including GLUT1, HK1, GPI, PFKFB3, PFK1, 
ALDOA, GAPDH, PGK1, ENO1, PDK1, LDHA, and LDHB (Fig. 5a, b). The mRNA levels of these glycolytic genes were moderately reduced in ADORA2A knockdown HRMECs under normoxia compared with those of control HRMECs. Under hypoxia conditions, ADORA2A knockdown remarkably downregulated mRNA levels of these glycolytic genes. Protein levels of
PFKFB3, a key glycolytic activator ${ }^{8,9}$, are also reduced in hypoxic HRMECs treated with siRNAs targeting human ADORA2A $\left(\mathrm{siA}_{2 \mathrm{~A}} \mathrm{R}\right.$, Supplementary Fig. 8) and OIR retinas from Adora $2 a^{V E C-K O}$ mice (Supplementary Fig. 9).To test whether similar changes also take place in retinal vessels or ECs in vivo, we used laser-capture microdissection to isolate retinal vessels and anti-

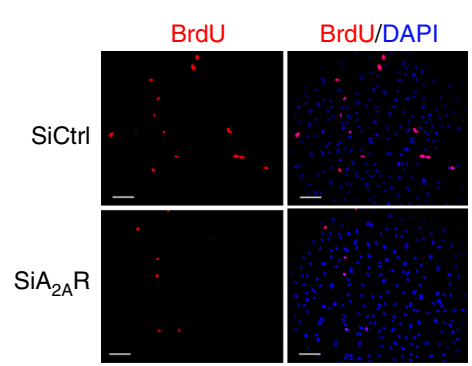

e

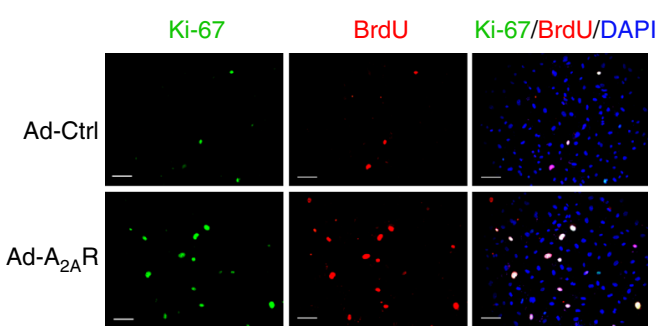

j

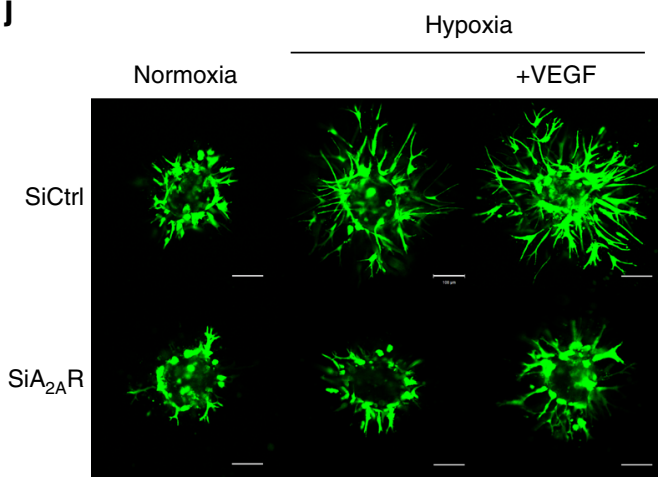

$\mathbf{p}$

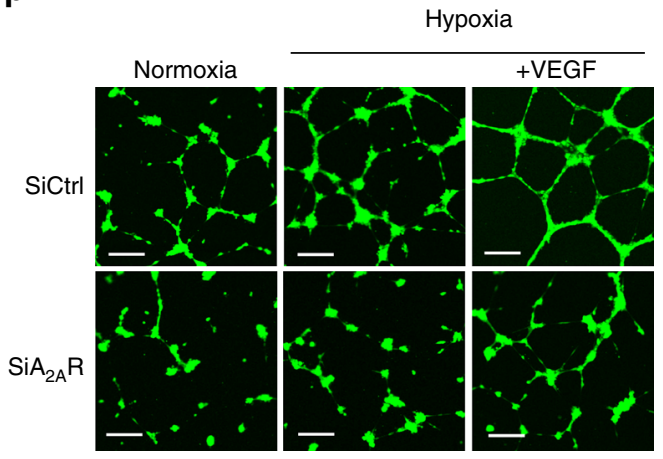

b
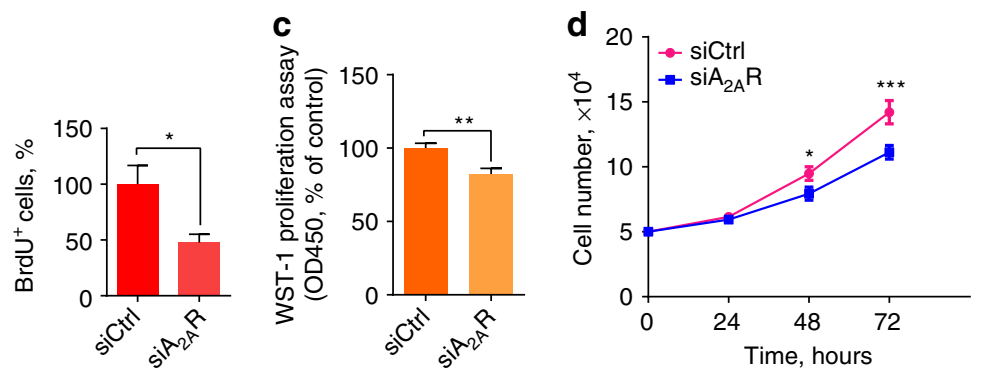

f

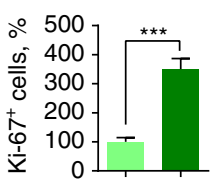

g

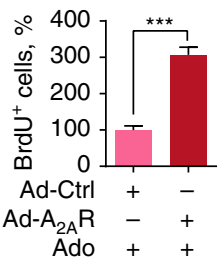

$\mathbf{k}$

h

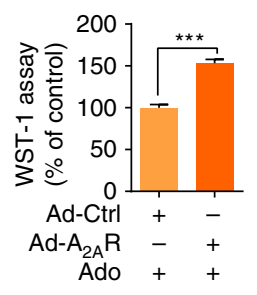

i

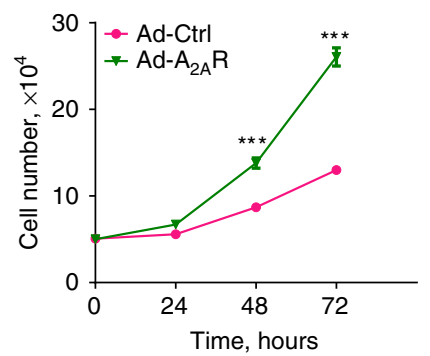

m

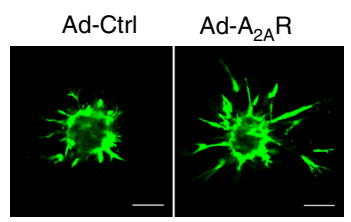

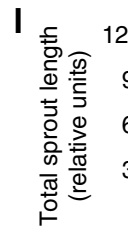
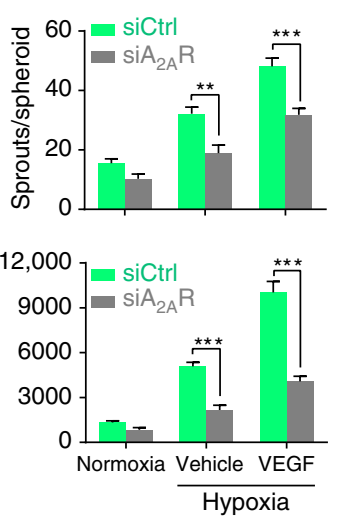

$\mathbf{q}$
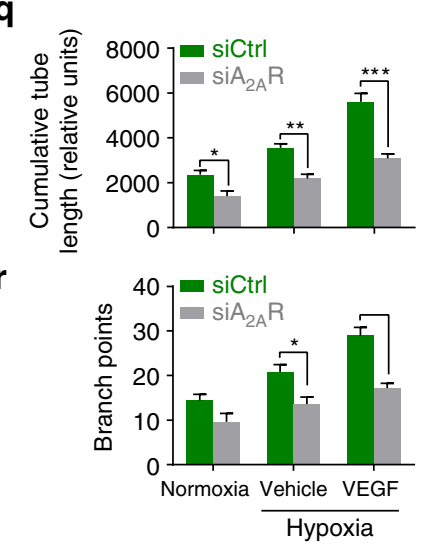
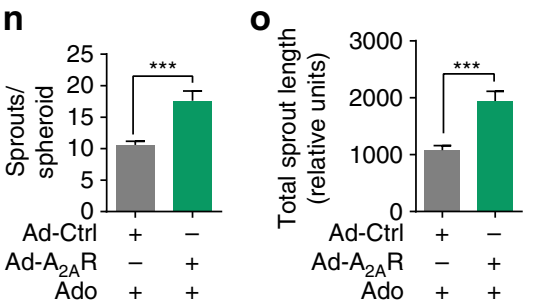

S

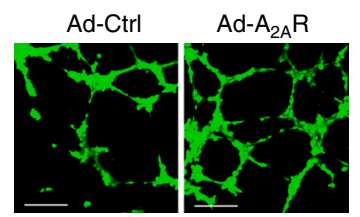

$\mathbf{u}$

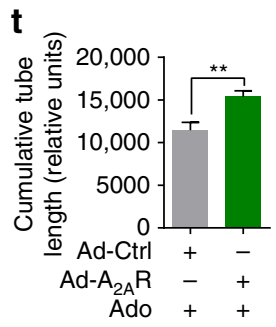

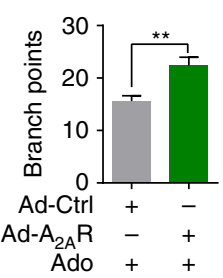


CD31 antibody-conjugated magnetic beads to isolate mouse retinal ECs from Adora $2 a^{V E C-K O}$ and control mice, respectively, and analyze gene expression specifically in vessels or ECs by RealTime PCR. Consistent with the findings from the in vitro data, most of the key glycolytic genes were significantly downregulated in vessels (Fig. 5c) or ECs (Supplementary Fig. 10) isolated from the OIR retinas of Adora2a ${ }^{V E C-K O}$ mice compared with controls.

Lactate is the metabolite generated in the glycolytic pathway by lactate dehydrogenase A (LDHA) and functions as a signaling molecule for angiogenesis ${ }^{10,22}$. Hypoxia resulted in a robust increase in the level of secreted lactate by HRMECs, but this increase was attenuated by transfection of ADORA2A siRNA into HRMECs (Fig. 5d). Using Seahorse Flux analysis, we further assessed the glycolytic function of HRMECs directly by measuring the extracellular acidification rate (ECAR). As shown in Fig. 5e, f, ADORA2A knockdown in HRMECs significantly reduced glucose-induced glycolysis and also reduced maximal glycolytic capacity. Basal cellular oxygen consumption (OCR), indicative of mitochondrial respiratory activity, and ATP production were not affected by ADORA2A deletion (Supplementary Fig. 11a, b) or overexpression (Supplementary Fig. 11c, d), whereas maximal respiration and spare respiratory capacity were decreased in $\mathrm{Ad}-\mathrm{A}_{2 \mathrm{~A}} \mathrm{R}$ plus adenosine-treated cells. Altogether, these results demonstrate that ADORA2A is an endogenous regulator of glycolysis in retinal ECs.

We next explored whether ADORA2A activation per se without hypoxia is able to increase EC glycolysis. ADORA2A overexpression or adenosine treatment alone resulted in a modest increase in expression of key glycolytic genes, lactate production and ECAR in HRMECs (Fig. 5g-j). Interestingly, these increased parameters were strikingly further elevated when adenosine was added to ADORA2A-overexpressing HRMECs compared with those from control cells or cells treated with adenosine alone (Fig. 5g-j), supporting that both ADORA2A upregulation and adenosine availability are crucial for HRMEC glycolysis.

To further confirm the findings obtained through genetic approaches, we also used the ADORA2A antagonist ZM241385 and ADORA2A agonist CGS21680 to block or activate ADORA2A in HRMECs, respectively. As shown in Supplementary Fig. 12, the levels of secreted lactate were decreased in ZM241385-treated cells and increased in CGS21680-treated cells compared with vehicle treated controls under hypoxic conditions.

\footnotetext{
ADORA2A-mediated glycolysis in HRMEC angiogenesis in vitro. After having shown that ADORA2A plays a crucial role in HRMEC glycolysis, we examined whether inhibition of glycolysis could block the ADORA2A-mediated angiogenic effects. As expected, ADORA2A overexpression induced HRMEC proliferation and subsequent sprouting in the presence of
}

adenosine (Fig. 6a-g). Blockade of glycolysis by the PFKFB3 inhibitor 3-(3-pyridinyl)-1-(4-pyridinyl)-2-propen-1-one (3PO) or the non-metabolizable glucose analog 2-deoxy-D-glucose (2-DG) significantly reversed the ADORA2A-mediated pro-angiogenic effect. We further examined if glycolysis blockade is able to inhibit HRMEC spheroid sprouting when ADORA2A is activated by hypoxia. Indeed, 3PO or 2-DG treatment dramatically decreased hypoxia-mediated hypersprouting (Fig. $6 \mathrm{~h}-\mathrm{j})$. Overall, these data suggest that ADORA2A-mediated glycolysis has a critical role in the ADORA2A activation-driven angiogenic response.

ADORA2A and glycolysis in the formation of endothelial tips. Vascular growth is led by endothelial tip cells and supported by proliferative stalk cells, and each of these cell types is signatured with an array of unique genes ${ }^{23}$. To examine whether ADORA2A and glycolysis modulate the formation of endothelial tip cells, we measured the expression of these signature genes. In HRMECs, ADORA2A knockdown downregulated the tip-cell-enriched genes (CXCR4, CD34, and VEGFA), and upregulated the stalk-cell-enriched genes (HEY1, HEY2, NTN4, and DLL4) under baseline conditions (Fig. 7a). Blocking of Notch signaling using the $\gamma$-secretase inhibitor DAPT ( $N$-[N-(3,5-difluorophenacetyl)-Lalanyl]-S-phenylglycine t-butylester) promoted a tip cell phenotype $^{24}$. Indeed, DAPT treatment caused an expected increase in the expression of tip-cell-enriched genes and downregulation of stalk-cell-specialized genes, whereas ADORA2A knockdown (Fig. 7a) or 2-DG-mediated glycolysis blockade (Fig. 7b) counteracted the DAPT-mediated alteration of the genetic tip vs. stalk cell signature upon Notch signaling blockade. Accordingly, pharmacological inhibition of Notch signaling in HRMECs has been shown to increase the number and length of sprouts, but this hyper-sprouting was abolished when cells were treated with ADORA2A siRNA ( $\mathrm{siA}_{2 \mathrm{~A}} \mathrm{R}$, Fig. $7 \mathrm{c}, \mathrm{d}$ ). Using a model of mosaic spheroids, we further assessed whether ADORA2A knockdown would influence tip cell formation induced by Notch signaling inhibition. HRMECs were infected with adenovirus encoding mCherry (red fluorescence) or EGFP (green fluorescence), then transfected with either siCtrl (control) or $\operatorname{siA}_{2 \mathrm{~A}} \mathrm{R}$. The consequent siCtrl ${ }^{\mathrm{GFP}}$, siCtrl ${ }^{\mathrm{RED}}$, or $\mathrm{siA}_{2 \mathrm{~A}} \mathrm{R}^{\mathrm{RED}}$ cells were mixed in a $1: 1$ ratio to generate mosaic spheroids. In control spheroids, generated by mixing siCtrl ${ }^{\mathrm{RED}}$ and siCtrl ${ }^{\mathrm{GFP}}$ cells, a comparable fraction of siCtrl ${ }^{\text {FFP }}$ and siCtrl ${ }^{\text {RED }}$ ECs were observed at the tip position (Fig. 7e, f). In contrast, in spheroids containing a 1:1mixture of $\mathrm{siA}_{2 \mathrm{~A}} \mathrm{R}^{\mathrm{RED}}$ and siCtrl GFP cells in the presence of DAPT, a reduced number of $\mathrm{siA}_{2 \mathrm{~A}} \mathrm{R}^{\mathrm{RED}} \mathrm{ECs}$ was identified at the tip. Similar results were obtained with HRMECs under hypoxic conditions (Fig. 7g, h).

Fig. 4 ADORA2A regulates HRMEC proliferation, sprouting and tube formation. a, b Bromodeoxyuridine (BrdU) staining of HRMECs transfected with siRNAs targeting human $A D O R A 2 A\left(\mathrm{si}_{2 \mathrm{~A}} \mathrm{R}\right)$ or with a non-targeting negative control $\left(\mathrm{siC}\right.$ trl) under hypoxia conditions $\left(0.5 \% \mathrm{O}_{2}\right) . n=6 .{ }^{\star} P<0.05$. c Cell proliferation measured by WST-1 cell proliferation assay. $n=6$. ${ }^{\star \star} P<0.01$. d Growth curves of transfected cultures. $n=6 .{ }^{\star} P<0.05,{ }^{\star \star \star} P<0.001$ vs. siCtrl. e-g Ki-67 and BrdU staining of HRMECs. HRMECs were infected with a recombinant adenovirus vector expressing human $A D O R A 2 A\left(A d-A_{2 A} R\right.$ ) or a negative control adenovirus (Ad-Ctrl) in the presence of adenosine. Scale bars: $50 \mu \mathrm{m} . n=6 .{ }^{\star \star \star} P<0.001$. $\mathbf{h}$ Cell proliferation measured by WST-1 cell proliferation assay. $n=6$. ${ }^{\star \star \star} P<0.001$. $\mathbf{i}$ Growth curves of HRMECs over $72 \mathrm{~h}$ following infection with Ad-Ctrl or Ad- $A_{2 A} R$ in the presence of adenosine. $n=6$. ${ }^{\star \star \star} P<0.001$ vs. Ad-Ctrl. $\mathbf{j}-\mathbf{u} H R M E C s$ were transfected with si $A_{2 A} R$ or siCtrl, or infected with Ad-Ctrl or Ad- $A_{2 A} R$, and then were cultured in collagen gel to grow into 3D multicellular spheroids, or on a 2D matrix to form a tube network in the presence or absence of VEGF or adenosine. $\mathbf{j}$, $\mathbf{m}$ Representative images of spheroidal sprouting after culturing for $24 \mathrm{~h}$ in collagen matrix under hypoxia $\left(0.5 \% \mathrm{O}_{2}\right)$ or normoxia $\left(21 \% \mathrm{O}_{2}\right)$. Scale bars: $100 \mu \mathrm{m}$.

Morphometric quantification of spheroid sprouting by calculating the number of sprouts per spheroid $\mathbf{k}, \mathbf{n}$ as well as total sprout length $\mathbf{I}, \mathbf{o}$. $n=10$ per group. $n$ is number of spheroids quantified. ${ }^{\star \star} P<0.01$; ${ }^{\star \star \star} P<0.001$. Representative fluorescence photographs of angiogenic tube formation $\mathbf{p}$, $\mathbf{s}$. Scale bars: $200 \mu \mathrm{m}$. Cumulative tube length quantified using the Image J software $\mathbf{q}, \mathbf{t}$, and branch points calculated from five experiments in each case $\mathbf{r}, \mathbf{u} .{ }^{\star} P<0.05 ;{ }^{\star \star} P<0.01 ;{ }^{\star \star \star} P<0.001$. Data are represented as means \pm s.e.m. Statistical significance was determined by unpaired Student's $t$-test (for $\mathbf{b}, \mathbf{c}, \mathbf{f}, \mathbf{g}, \mathbf{h}, \mathbf{k}, \mathbf{I}, \mathbf{n}, \mathbf{0}, \mathbf{q}, \mathbf{r}, \mathbf{t}, \mathbf{u}$ ) and two-way ANOVA followed by Bonferroni test (for $\mathbf{d}, \mathbf{i}$ ) 
ADORA2A activation induces HRMEC glycolysis via HIF-1 $\alpha$. HIF-1 is the principal regulator of the transcriptional response to hypoxia. Almost all enzymes of the glycolytic cascade, as well as glucose transport proteins, are upregulated by HIF- $1 \alpha^{25}, 26$. Therefore, we hypothesized that HIF- $1 \alpha$ might mediate the regulation effect of ADORA2A on EC glycolysis. To test this, we first analyzed the effects of $A D O R A 2 A$ knockdown or overexpression on HIF- $1 \alpha$ expression in HRMECs. We found that HIF- $1 \alpha$ mRNA levels in HRMECs were indistinguishable between control and $A D O R A 2 A$ knockdown or overexpression groups
(Supplementary Fig. 13). In contrast, evaluation of HIF-1 $\alpha$ protein expression in $\mathrm{siA}_{2 \mathrm{~A}} \mathrm{R}$ or $\mathrm{Ad}-A_{2 A} R$ - infected HRMECs by western blot showed reduced or increased HIF-1 $\alpha$ protein levels, respectively (Fig. 8a-d). Consistent with these observations, ECspecific $A D O R A 2 A$ deletion decreased the expression of HIF-1 $\alpha$ in retinal ECs of OIR mice at P15 (initial stages of neovessel formation) (Fig. 8e, f). Furthermore, we examined the role of HIF- $1 \alpha$ in ADORA2A activation-mediated upregulation of glycolytic genes in HRMECs. Both hypoxia and Ad- $A_{2 A} R /$ adenosine-driven ADORA2A activation upregulated the key glycolytic

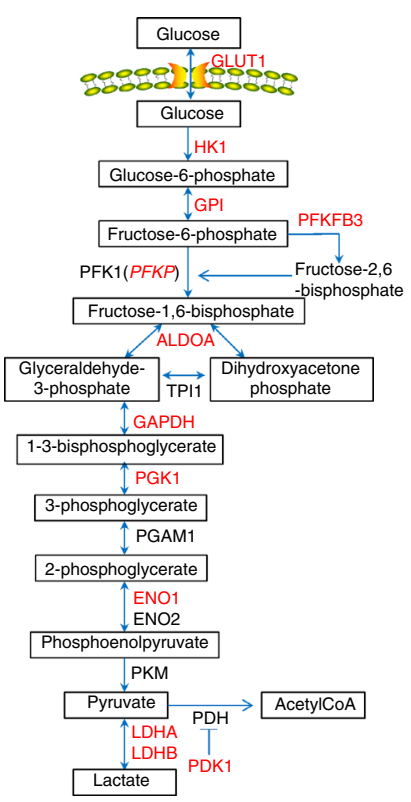

b

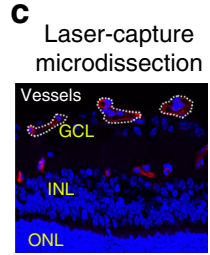

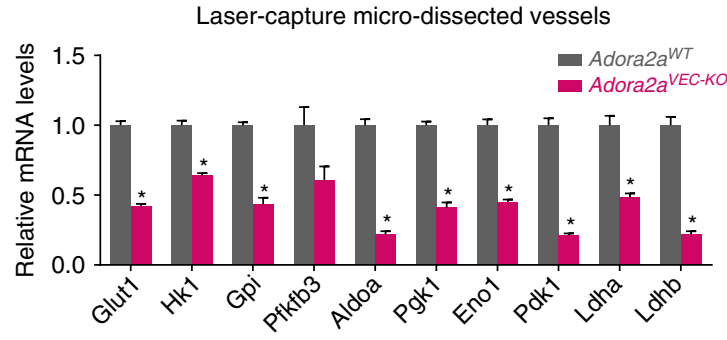

d

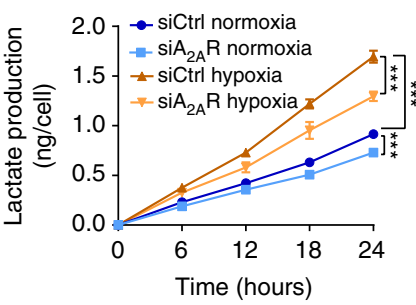

e

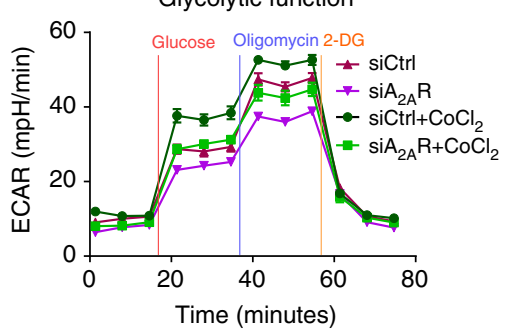

f

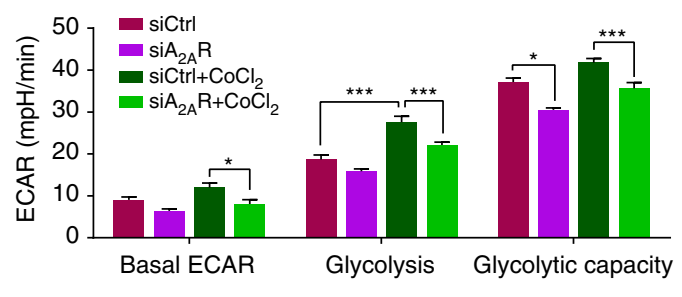

g

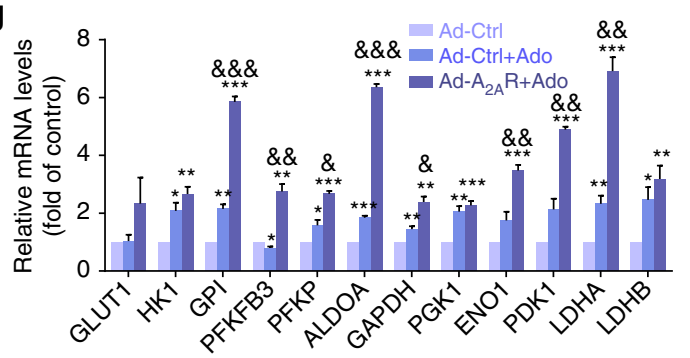

h

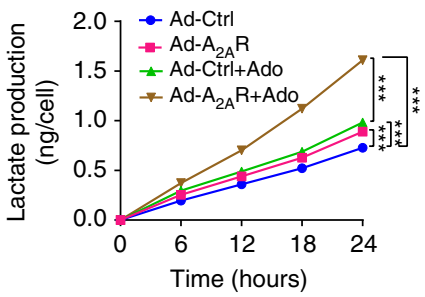

i

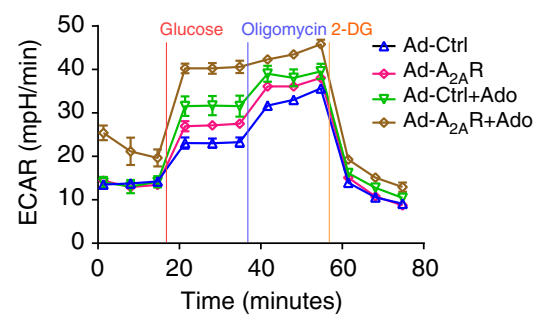

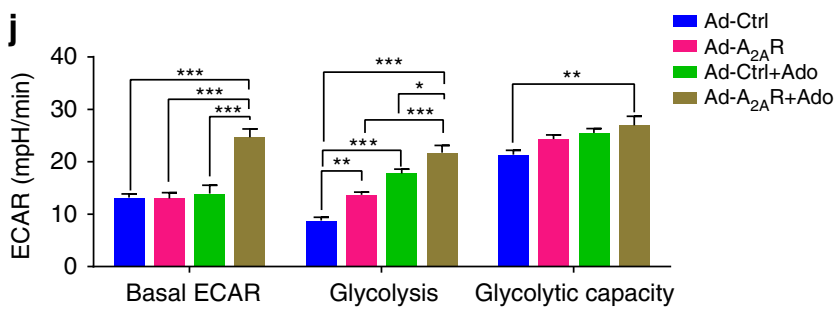


enzymes (Fig. 8g, h) and enhanced glycolytic function (Fig. 8i, j) in HRMECs. These effects were abolished by siHIF- $1 \alpha$ or treatment with the specific HIF inhibitor CAY10585. Altogether, these data demonstrate that activation of ADORA2A promotes glycolysis in HRMECs by enhancing HIF- $1 \alpha$ protein accumulation without altering its mRNA level.

ADORA2A regulates HIF-1 $\alpha$ level via translational pathways. In view of our findings that ADORA2A activation upregulates the protein level of HIF-1 $\alpha$ without affecting transcriptional induction of HIF-1 $\alpha$, we next explored whether ADORA2A regulates HIF- $1 \alpha$ translation or protein stability. To assess the effect of ADORA2A on HIF-1 $\alpha$ mRNA translation, the proteasome inhibitor MG132 was used to avoid the degradation of HIF- $1 \alpha$ protein. ADORA2A knockdown resulted in a much slower rate of MG132-induced HIF- $1 \alpha$ accumulation (Fig. 9a, c), indicating that HIF-1 $\alpha$ protein synthesis in HRMECs is markedly impaired by $A D O R A 2 A$ knockdown. To test the effect of ADORA2A on HIF- $1 \alpha$ protein stability, the protein translation inhibitor cycloheximide $(\mathrm{CHx})$ was employed to prevent de novo HIF- $1 \alpha$ protein synthesis. In the presence of $\mathrm{CHx}$, HIF- $1 \alpha$ levels rapidly declined in HRMECs under hypoxia and ADORA2A overexpression did not modify the degradation rate of HIF- $1 \alpha$ (Fig. 9b, d). Thus, ADORA2A modulates the protein synthesis of HIF- $1 \alpha$ but not its degradation.

To characterize the underlying mechanisms by which ADORA2A regulates HIF- $1 \alpha$ protein synthesis, we first surveyed several MAPK and PI3K/Akt signaling pathways. As shown in Fig. 9e, ADORA2A knockdown or adenosine treatment did not affect p38 kinase or JNK phosphorylation. However, we observed a profound activation of the ERK1/2 and Akt signaling pathways in HREMCs after adenosine treatment under hypoxia, and this activation was markedly reduced by ADORA2A knockdown. Furthermore, gain-of-function studies revealed more robust ERK1/2 and Akt phosphorylation after adenosine treatment in Ad-ADORA2A-infected HREMCs (Fig. 9f). These observations prompted us to hypothesize that ERK1/2 and Akt activation may govern the effects of ADORA2A activation on the enhancement of HIF- $1 \alpha$ protein level. In fact, the MEK/ERK and PI3K/Akt signaling pathways have been demonstrated to be involved in HIF- $1 \alpha$ translation via functional activation of the translation initiation factors $\mathrm{p} 70^{\mathrm{S} 6 \mathrm{~K}}$ and eIF-4E in various cells ${ }^{27-29}$. Indeed, in parallel with the alteration of ERK1/2 and Akt phosphorylation, ADORA2A knockdown significantly inhibited basal and adenosine-mediated activation of $\mathrm{p} 70^{\mathrm{S} 6 \mathrm{~K}}$ and eIF-4E (Fig. 9e). In addition, treatment of HRMECs with adenosine and Ad- $\mathrm{A}_{2 \mathrm{~A}} \mathrm{R}$ effectively increased the protein level of HIF-1 $\alpha$ (Fig. 9f), as well as the phosphorylation of both $\mathrm{p} 70^{\mathrm{S} 6 \mathrm{~K}}$ and eIF-4E, which was inhibited by the MEK inhibitor U0126 or PI3K inhibitor
LY294002 (Fig. 9g). Collectively, these results suggest that ADORA2A regulates HIF-1 $\alpha$ protein synthesis through the MEK/ERK and PI3K/Akt pathways that activate the HIF-1 $\alpha$ translational machinery.

\section{Discussion}

In this study, we demonstrate a novel cellular and molecular mechanism whereby endothelial ADORA2A activation promotes pathological angiogenesis in the retina. Retinal hypoxia leads to the activation of HIF-2 $\alpha$, which enhances ADORA2A expression. Increased ADORA2A in retinal ECs, in turn, enhances the accumulation of HIF- $1 \alpha$ via a translational pathway. Elevated HIF- $1 \alpha$ is largely responsible for elevation of glycolytic enzymes as well as endothelial glycolysis. ADORA2A-mediated glycolysis critically contributes to retinal EC proliferation, sprouting and angiogenesis (Fig. 10). These findings provide new insights into a previously unrecognized effect of ADORA2A on endothelial glycolysis in ischemic retinopathies and highlight the translational potential of targeting ADORA2A in the treatment of vision-threatening eye diseases.

Extracellular adenosine accumulates during hypoxia conditions and signals through four G-protein-coupled adenosine receptors (ADORA1, ADORA2A, ADORA2B, and ADORA3) ${ }^{12}$. Adenosine signaling events play a key role in various ischemic diseases, including ischemic proliferative retinopathies. In a previous study, Adora2a global knockout mice exhibited markedly suppressed retinal angiogenesis in the OIR model, which was accompanied by a low level of VEGF mRNA in the retina ${ }^{30}$. The critical role of VEGF in retinal angiogenesis has been long established by a large body of experimental studies and clinical application $^{31}$, 32. EC-autonomous VEGF is indispensable for vascular homeostasis while VEGF produced by non-ECs is critical for the angiogenic cascade ${ }^{33}$. Therefore, the low level of VEGF mRNA in Adora2a-deficient retinas may be likely due to Adora2a deficiency in non-ECs, and the Adora2a deficiency in non-ECs may mainly account for the suppressed retinal angiogenesis in Adora2a-deficient mice. Adora2a in macrophages has been reported to be highly involved in VEGF production ${ }^{34}$. We suspected that Adora $2 a$ deficiency in macrophages may be the major reason for the low level of VEGF and decreased neovascularization in the retina. However, Adora2a deficiency in myeloid cells, although decreasing the avascular area, did not significantly suppress neovascularization in OIR retinas. In contrast, endothelial Adora2a deficiency dramatically decreased both avascular and neovascularization areas in retinas of OIR models, suggesting that endothelial Adora2a is critical for pathological retinal angiogenesis. Since endothelial autocrine VEGF signaling is dispensable in angiogenesis in vivo $^{33}$, decreased VEGF production from Adora2a-deficient retinal ECs

Fig. 5 Adenosine-ADORA2A signaling regulates glycolysis in HRMECs and mouse retinal ECs. a Scheme showing the glycolytic pathway and associated enzymes. b Real-Time PCR analysis of the mRNA levels of glycolytic genes in HRMECs transfected with si $\mathrm{A}_{2 \mathrm{~A}} \mathrm{R}$ or siCtrl under normoxia $\left(21 \% \mathrm{O}_{2}\right.$ ) or hypoxia $\left(0.5 \% \mathrm{O}_{2}\right) . n=5 .{ }^{\star} P<0.05,{ }^{\star \star} P<0.01,{ }^{\star \star \star} P<0.001$ vs. siCtrl normoxia group; $\& P<0.05, \& \& p<0.01, \& \& \& P<0.001$ vs. siCtrl hypoxia group. c Real-Time PCR analysis of the mRNA levels of glycolytic genes in retinal blood vessels isolated with laser-capture microdissection from OIR-Adora2 $a^{W T}$ and Adora2a ${ }^{V E C-K O}$ mice at P17. $n=4$. ${ }^{\star} P<0.05$ vs. Adora2aWT group. $\mathbf{d}$ Levels of secreted lactate of HRMECs transfected with si $\mathrm{A}_{2 \mathrm{~A}} \mathrm{R}$ or $\mathrm{siCtrl}$ under normoxia or hypoxia for indicated times. $n=3$. ${ }^{\star \star \star} P<0.001$. e ECAR profile showing glycolytic function in siCtrl- and siA ${ }_{2 A} R$-transfected cells under hypoxia $\left(0.5 \% \mathrm{O}_{2}\right)$ or normoxia $\left(21 \% \mathrm{O}_{2}\right)$. Vertical lines indicate the time of addition of glucose $(10 \mathrm{mmol} / \mathrm{l})$, oligomycin $(2 \mu \mathrm{mol} / \mathrm{I})$, and $2-\mathrm{DG}(50 \mathrm{mmol} / \mathrm{I})$. f Quantification of glycolytic function parameters from e. $n=8$ for normoxic groups and $n=16$ for each of $\mathrm{CoCl}_{2}$ treatment groups. ${ }^{\star} P<0.05$; ${ }^{\star \star \star} P<0.001$. g Real-Time PCR analysis of the mRNA levels of glycolytic genes in HRMECs infected with Ad-Ctrl or Ad- $A_{2 A} R$, with or without adenosine treatment. $n=4$. ${ }^{\star} P<0.05 ;{ }^{\star \star} P<0.01 ;{ }^{\star \star \star} P<0.001$ vs. Ad-Ctrl; ${ }^{\&} P<0.05 ; \& \& P<0.01 ; \& \& \& P<0.001$ vs. Ad-Ctrl + Ado. h Levels of secreted lactate of HRMECs infected with $\mathrm{Ad}-\mathrm{A}_{2 \mathrm{~A}} \mathrm{R}$ or $\mathrm{Ad}-\mathrm{Ctrl}$ with or without adenosine treatment. $n=3$. ${ }^{\star \star \star} P<0.001 \mathrm{vs}$. Ad-Ctrl. $\mathbf{i}$ ECAR profile showing glycolytic function in Ad-Ctrl- and $\mathrm{Ad}-\mathrm{A}_{2 \mathrm{~A}} \mathrm{R}$-infected cells, with or without adenosine treatment. $\mathbf{j}$ Quantification of glycolytic function parameters from $\mathbf{i}$. $n=8$ per group.

${ }^{\star} P<0.05 ;{ }^{\star \star} P<0.01 ;{ }^{\star \star \star} P<0.001$ vs. Ad-Ctrl. Data are represented as means \pm s.e.m. Statistical significance was determined by unpaired Student's $t$-test (for $\mathbf{b}, \mathbf{c}, \mathbf{g}$ ), one-way ANOVA followed by Bonferroni test (for $\mathbf{f}, \mathbf{j}$ ), and two-way ANOVA followed by Bonferroni test (for $\mathbf{d}, \mathbf{h}$ ) 
a

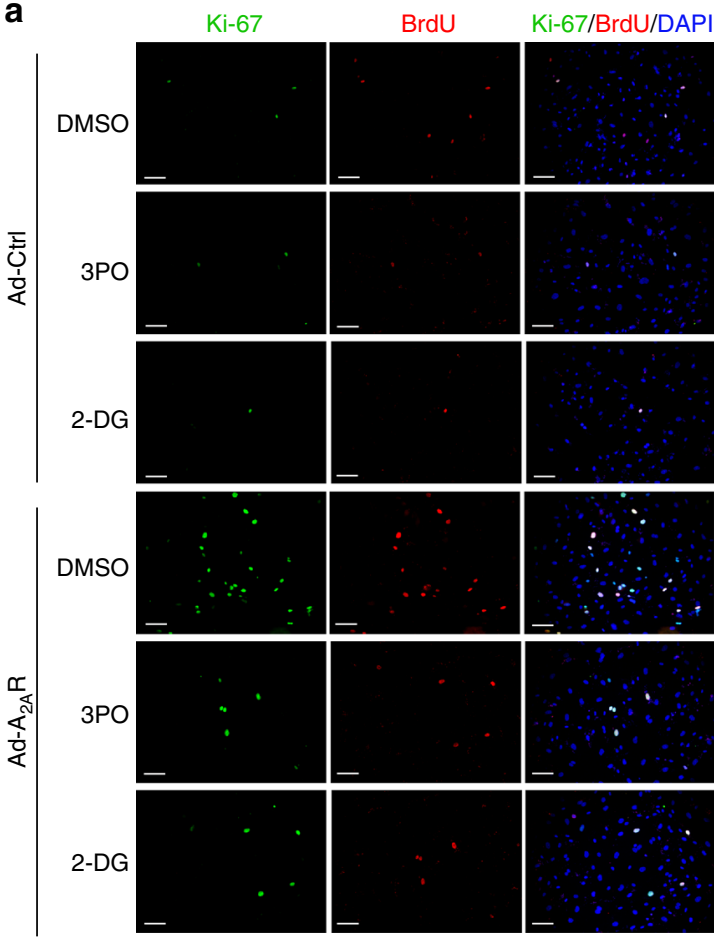

e

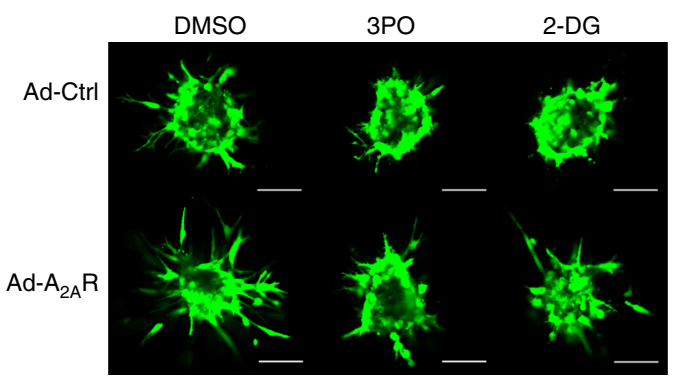

h

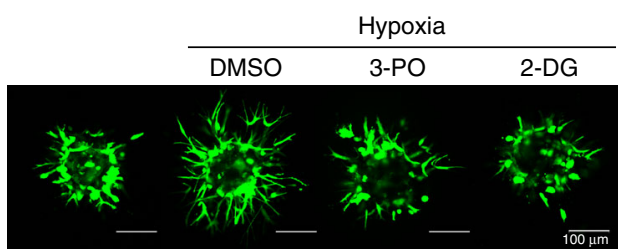

b

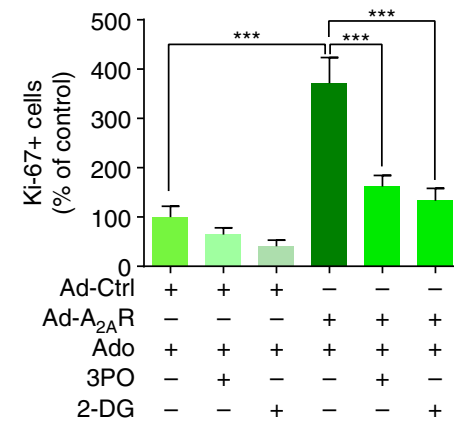

d
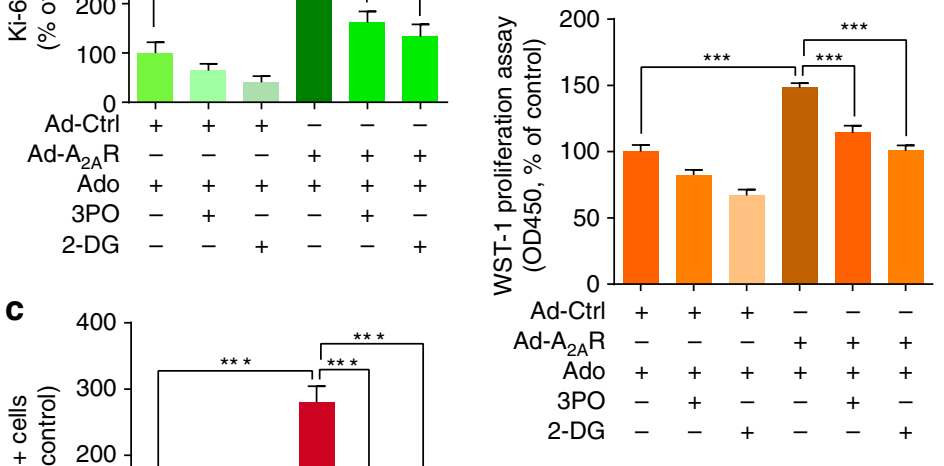

f

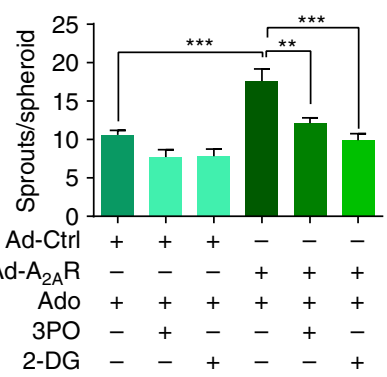

i

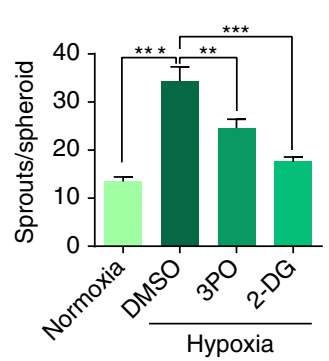

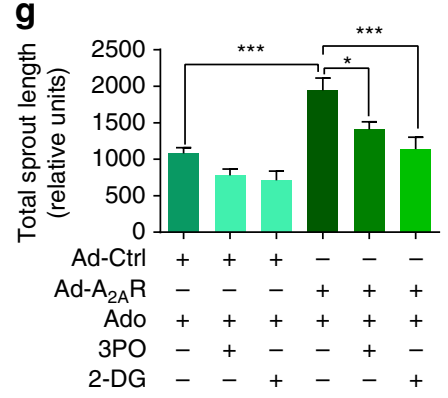

j

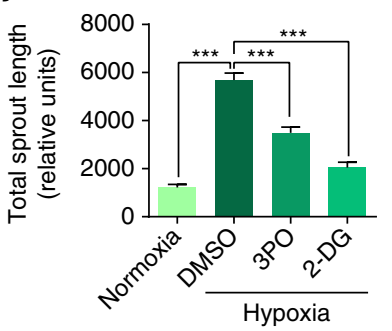

Fig. 6 Glycolysis is involved in ADORA2A activation-mediated HRMEC proliferation and sprouting. a-g Results of Ki-67 and BrdU staining a-c, WST-1 cell proliferation assay $\mathbf{d}$ and EC spheroid sprouting assay $\mathbf{e}-\mathbf{g}$ in HRMECs. HRMECs were infected with Ad-Ctrl or Ad- $A_{2 A} R$ adenoviruses, and then treated with adenosine in the presence or absence of two different glycolytic inhibitors (3PO, $10 \mu \mathrm{M}$, and 2-DG, $5 \mathrm{mM}) . n=6 .{ }^{\star} P<0.05 ;{ }^{\star \star} P<0.01 ;{ }^{\star \star \star} P<0.001$. Scale bars: $50 \mu \mathrm{m}$ for a; Scale bars: $100 \mu \mathrm{m}$ for e. h-j HRMECs were cultured as 3D multicellular spheroids in the presence or absence of 3PO or 2DG. Representative images of spheroidal sprouting after culturing for $24 \mathrm{~h}$ in collagen matrix under hypoxia $\left(0.5 \% \mathrm{O}_{2}\right)$ or normoxia $\left(21 \% \mathrm{O}_{2}\right) \mathbf{h}$. Morphometric quantification of spheroid sprouting by calculating the number of sprouts per spheroid $\mathbf{i}$ and total sprout length $\mathbf{j}$. $n=10$ per group ( $n$ is number of spheroids quantified). ${ }^{\star} P<0.05,{ }^{\star \star} P<0.01 ;{ }^{\star \star \star} P<0.001$. Scale bars: $100 \mu \mathrm{m}$. Data are represented as means \pm s.e.m. Statistical significance was determined by oneway ANOVA followed by Bonferroni test

may explain only in part the decreased pathological angiogenesis in retinas of the OIR model in endothelial Adora2a-deficient mice.

Tumor cells mainly rely on aerobic glycolysis, a phenomenon termed "the Warburg effect," to generate ATP and provide substances for fast growth ${ }^{35}$. Interestingly, vascular ECs use the same pathway to generate their energy. In ECs, glycolysis generates $85 \%$ of the total $\mathrm{ATP}^{8}$. Of this, ECs use $60 \%$ for maintenance of homeostasis and $40 \%$ for proliferation 36,37 PFKFB3 knockdown markedly suppressed angiogenesis, demonstrating the importance of glycolysis for endothelial angiogenesis $^{8-10}$. Growth factors such as VEGF and FGF2 enhance the expression of the key glycolytic enzyme PFKFB3 and increase glycolysis to support the high ATP demands for 
a

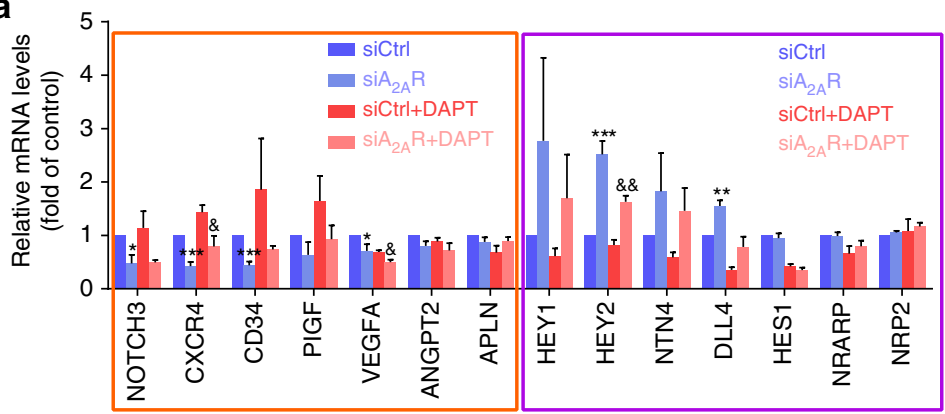

b

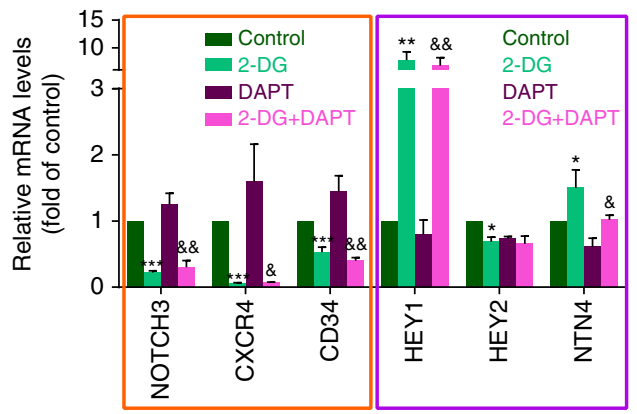

C

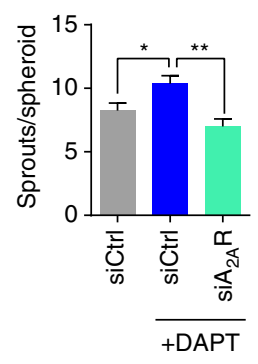

d

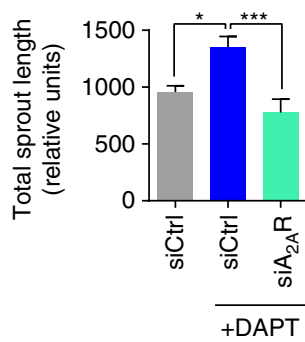

e

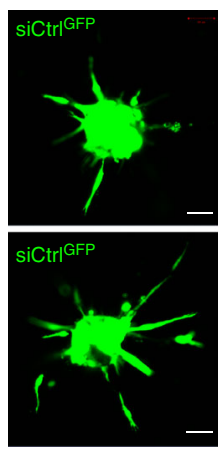

f

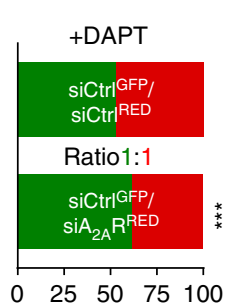

Tip cell contribution (\%)

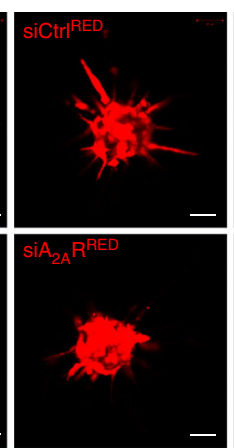

h

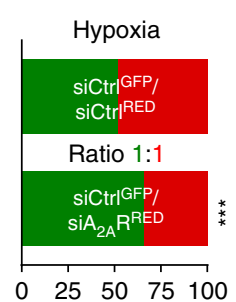

Tip cell contribution (\%)

\section{g}
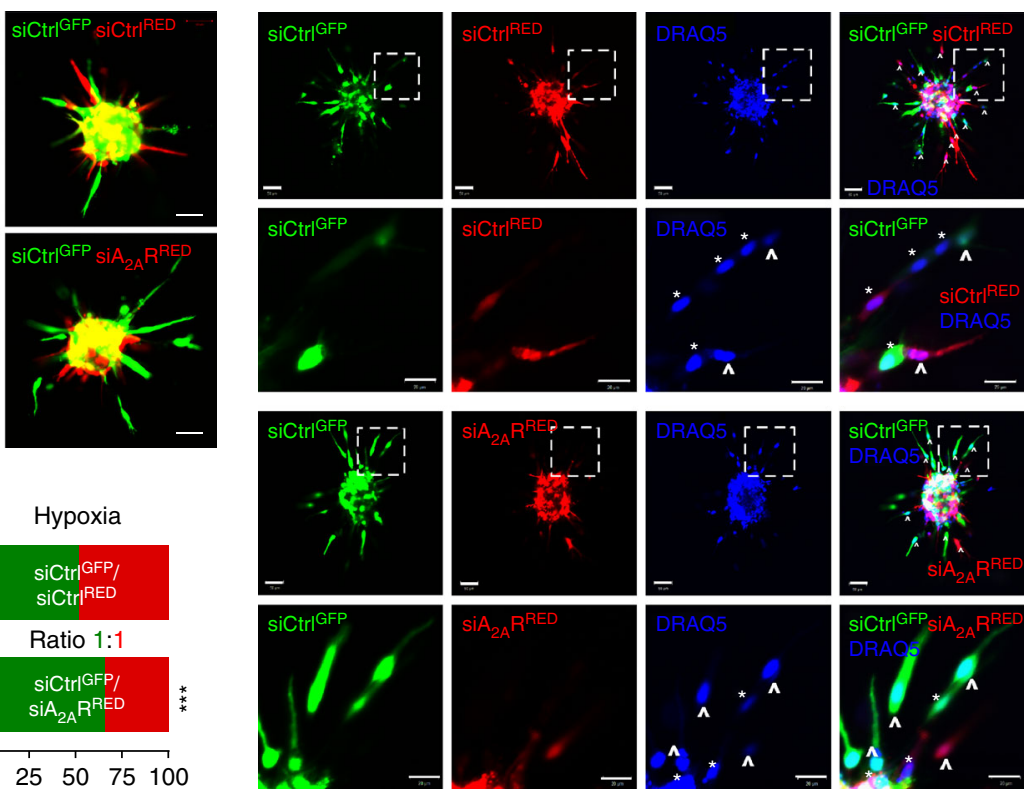

Fig. 7 ADORA2A regulates HRMEC tip cell formation. a Real-Time PCR analysis of the mRNA levels of the sprouting-governing genes in HRMECs transfected with $\mathrm{siA}_{2 \mathrm{~A}} \mathrm{R}$ or siCtrl, in the presence or absence of DAPT $\left(n=3 ;{ }^{\star} P<0.05 ;{ }^{\star \star} P<0.01 ;{ }^{\star \star \star} P<0.001 \mathrm{vs}\right.$. siCtrl; $\& P<0.05, \& \& P<0.01 \mathrm{vs}$. siCtrl + DAPT). $\mathbf{b}$ Real-Time PCR analysis of the mRNA levels of the sprouting-governing genes in 2-DG-treated HRMECs in the presence or absence of $\operatorname{DAPT}\left(n=4 ;{ }^{\star} P<0.05 ;{ }^{\star \star} P<0.01 ;{ }^{\star \star \star} P<0.001\right.$ vs. vehicle control; $\& P<0.05, \& \& P<0.01$ vs. DAPT). c, d Morphometric quantification of spheroid sprouting from cells transfected with siCtrl or $\mathrm{si}_{2 \mathrm{~A}} \mathrm{R}$ in the presence or absence of DAPT. $n=10$ per group. $n$ is number of spheroids quantified. ${ }^{\star} P<0.05$; ${ }^{\star \star} P<0.01$; ${ }^{* \star} P<<0.001$. e Representative fluorescence photographs of EC spheroids containing a 1:1 mixture of siCtrl ${ }^{\text {GFP }}$ and siCrl ${ }^{R E D}$ ECs, or a 1:1 mixture of siCtrl ${ }^{G F P}$ ECs and ECs with siA $A_{2 A} R^{R E D}$ in the presence of DAPT. Scale bars, $100 \mu \mathrm{m}$. $\mathbf{f}$ Quantification of the fraction of tip cells with the indicated genotypes shown in $\mathbf{e}\left(n=10 ;{ }^{\star \star \star} P<0.001\right.$ vs. siCtrl $\left.{ }^{\text {GFP }}\right)$. $\mathbf{g}$ Representative fluorescence photographs of mosaic EC spheroids containing a 1:1 mixture of $\mathrm{siCtrl}^{\mathrm{GFP}}$ ECs and siA $\mathrm{A}_{2 \mathrm{~A}} \mathrm{R}^{\mathrm{RED}}$ ECs under hypoxia conditions. Cells were stained with DRAQ5 (blue) to mark EC nuclei. Tip cells are indicated by “^"; Stalk cells are indicated by "*^. The 2nd and 4th rows are magnification of the boxed regions in the 1st and 3rd rows, respectively. Scale bar: $50 \mu \mathrm{m}$ (1st and 3rd rows) and $20 \mu \mathrm{m}$ (2nd and 4th rows). $\mathbf{h}$ Quantification of the fraction of tip cells with the indicated genotypes shown in $\mathbf{g}\left(n=10\right.$ per group). ${ }^{\star \star \star} P<0.001$ vs. siCtrl ${ }^{G F P}$. Data are represented as means \pm s.e.m. Statistical significance was determined by unpaired Student's $t$-test (for $\mathbf{a}, \mathbf{b}, \mathbf{f}, \mathbf{h}$ ) and one-way ANOVA followed by Bonferroni test (for $\mathbf{c}, \mathbf{d}$ ) 
a

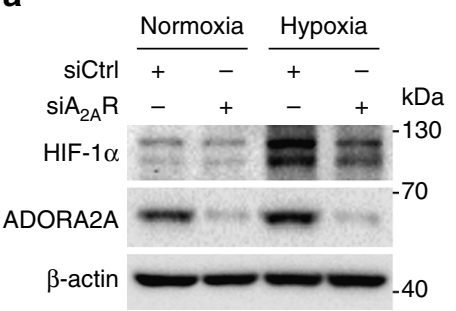

b

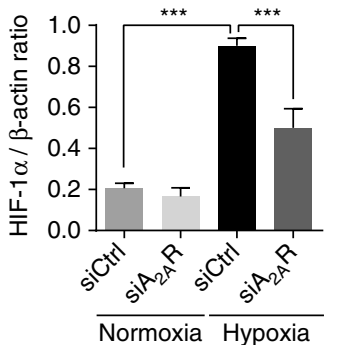

C

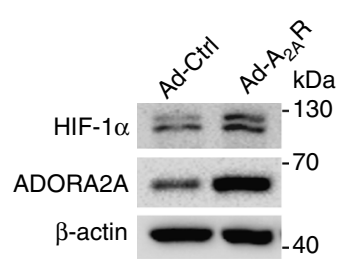

d

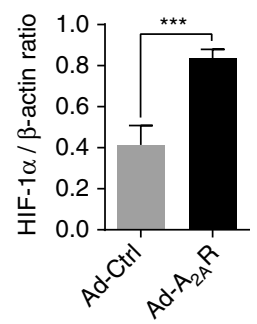

e
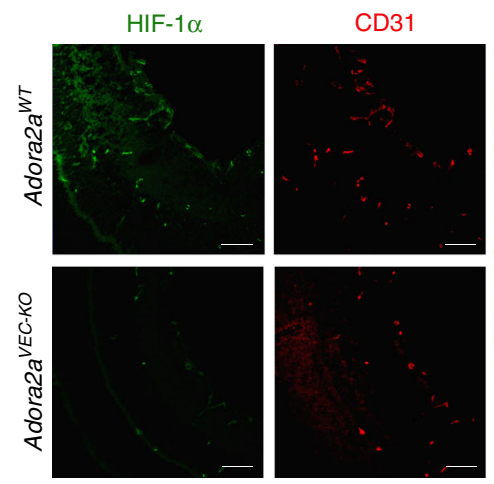

DAPI
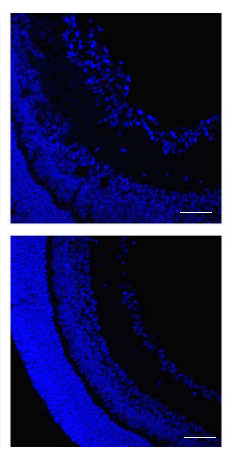

Merge

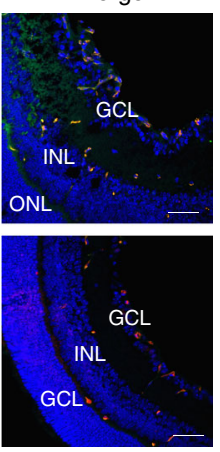

f

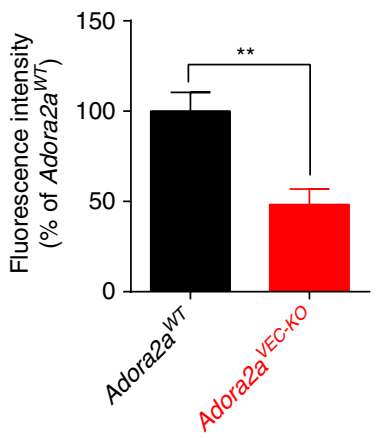

g

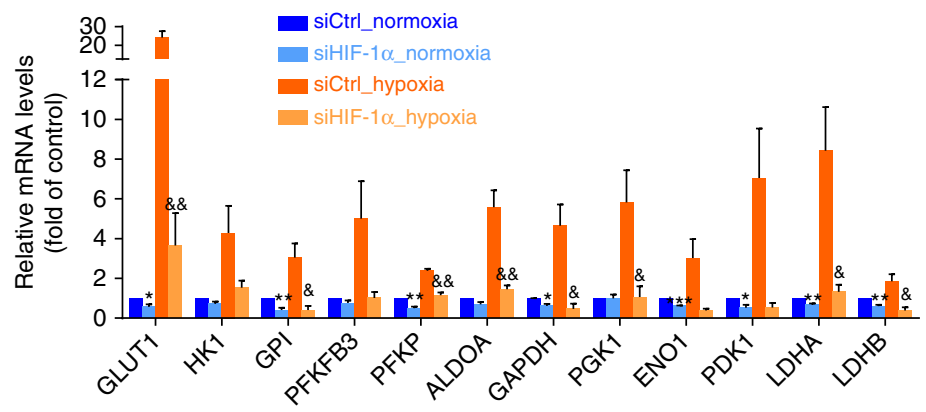

h

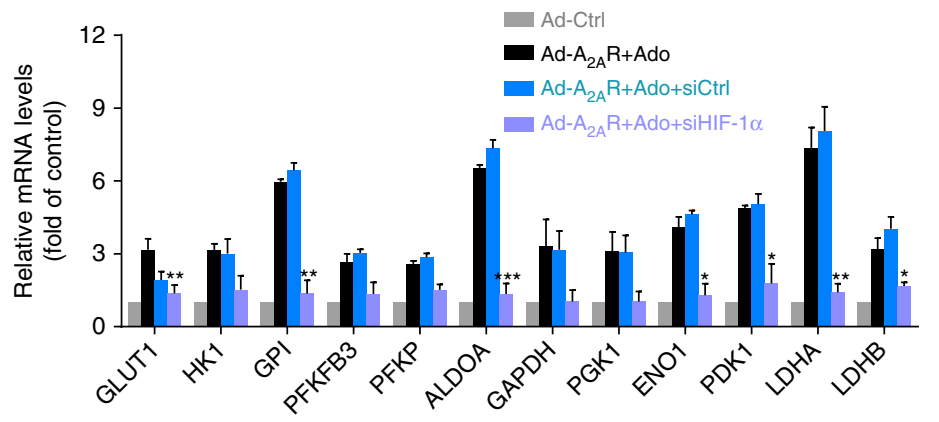

i

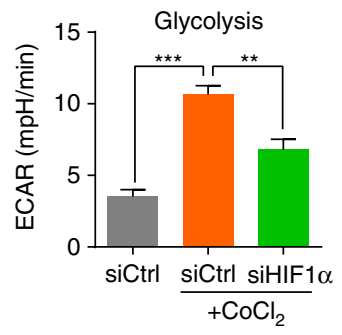

j

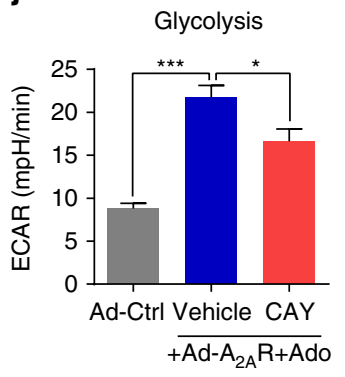

Fig. 8 ADORA2A activation mediates increase in glycolysis via a HIF-1 $\alpha$-dependent pathway. a-d Western blot analysis of HIF-1 $\alpha$ mRNA and protein expression in HRMECs transfected with si $\mathrm{A}_{2 \mathrm{~A}} \mathrm{R}$ or siCtrl under hypoxia $\left(0.5 \% \mathrm{O}_{2}\right)$ or normoxia $\left(21 \% \mathrm{O}_{2}\right) \mathbf{a}$, b, or infected with $\mathrm{Ad}-\mathrm{Ctrl}$ or $\mathrm{Ad}-\mathrm{A}_{2 \mathrm{~A}} \mathrm{R}$ under normoxia c, $\mathbf{d} . n=4 .{ }^{\star \star \star} P<0.001$. e, $\mathbf{f} \mathrm{HIF}-1 \alpha$ immunofluorescent staining of OIR retinas from Adora2 $a^{W T}$ and Adora2 $a^{V E C-K O}$ mice at postnatal day ( $\mathrm{P}$ ) 15 . Representative green (HIF-1 $\alpha$ ), red (CD31), blue (nuclei, DAPI), and merged images captured with confocal fluorescent microscopy. GCL ganglion cell layer, INL inner nuclear layer, ONL outer nuclear layer. Scale bar, $50 \mu \mathrm{m}$. The fluorescence intensity of HIF-1 $\alpha$ staining was calculated by Image J software then normalized to that of WT control (Adora2 $a^{W T}$ ). $n=6$ mice for each group. ${ }^{\star \star} P<0.01$. $\mathbf{g}$ Real-Time PCR analysis of the mRNA levels of glycolytic genes in HRMECs transfected with siHIF-1 $\alpha$ or siCtrl under normoxia $\left(21 \% \mathrm{O}_{2}\right)$ or hypoxia $\left(0.5 \% \mathrm{O}_{2}\right) . n=3$. ${ }^{\star} P<0.05$; ${ }^{\star \star} P<0.01$; ${ }^{\star \star \star} P<0.001$ vs. siCtrl normoxia group; $\& P<0.05$; \&\&P<0.01 vs. siCtrl hypoxia group. $\mathbf{h}$ Real-Time PCR analysis of the mRNA levels of glycolytic genes in HRMECs. Cells were transfected with HIF-1 $\alpha$ siRNA or siCtrl for $24 \mathrm{~h}$ under normoxia, and further infected with $\mathrm{Ad}-\mathrm{A}_{2 \mathrm{~A}} \mathrm{R}$ or $\mathrm{Ad}-\mathrm{Ctrl}$ for an additional $24 \mathrm{~h}$, followed by adenosine treatment for another $12 \mathrm{~h} . n=4 .{ }^{\star} P<0.05 ;{ }^{\star \star} P<0.01 ;{ }^{\star \star \star} P<0.001 \mathrm{vs}$. Ad- $\mathrm{A}_{2 \mathrm{~A}} \mathrm{R}+\mathrm{Ado}+\mathrm{siCtrl}$ group. i Quantification of glycolytic function in HRMECs transfected with siCtrl or siHIF-1 $\alpha$ in the presence of $\mathrm{CoCl}_{2}(200 \mu \mathrm{M}) . n=8$ per group. ${ }^{\star} P<0.05 ;{ }^{\star \star \star} P<0.001$. j Quantification of glycolytic function in HRMECs infected with Ad-Ctrl or $\mathrm{Ad}-\mathrm{A}_{2 \mathrm{~A}} \mathrm{R}$, with or without adenosine treatment, in the presence or absence of the HIF-1 $\alpha$ inhibitor CAY10585. $n=7,7,6$, respectively. ${ }^{\star} P<0.05 ;{ }^{\star \star \star} P<0.001$. Data are represented as means \pm s.e.m. Statistical significance was determined by unpaired Student's $t$-test (for $\mathbf{d}, \mathbf{f}, \mathbf{g}, \mathbf{h}$ ) and one-way ANOVA followed by Bonferroni test (for $\mathbf{b}, \mathbf{i}, \mathbf{j}$ ) 
a

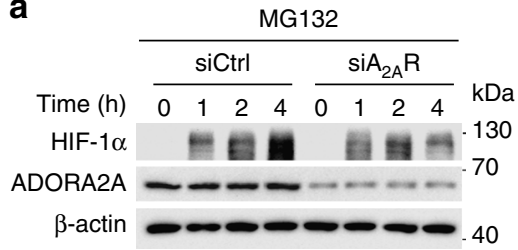

b

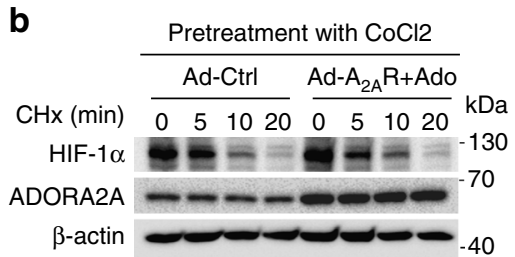

d

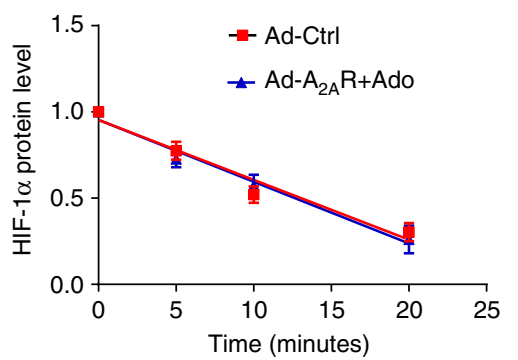

e
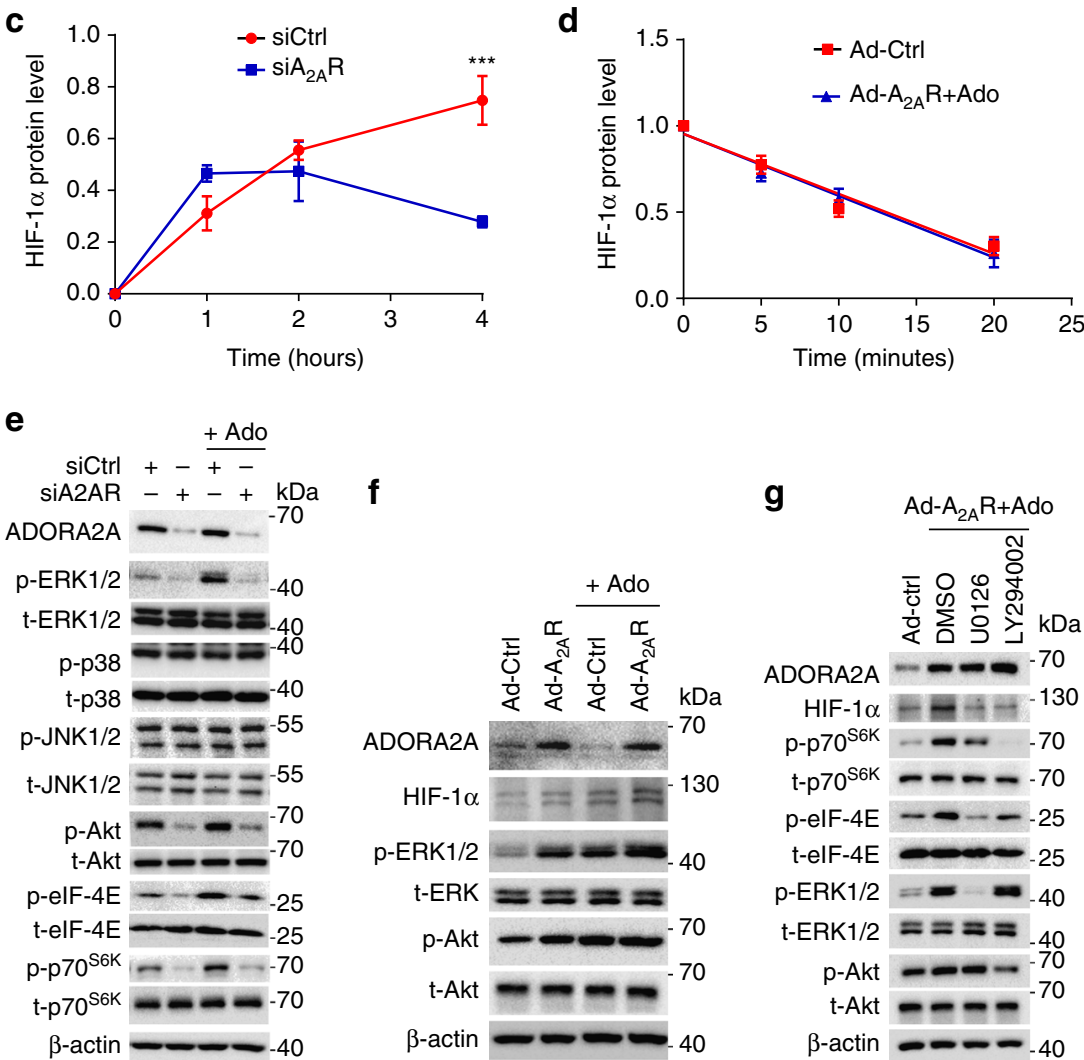

Fig. 9 ADORA2A regulates HIF-1 $\alpha$ protein level through activation of the PI3K/Akt and MEK/ERK-mediated translational machinery. a Western blot analysis of HIF- $1 \alpha$ at protein level in HRMECs. Cells were transfected with si $A_{2 A} R$ or the siCtrl for $48 \mathrm{~h}$, and then treated with MG-132 (10 $\mu$ M) for 1,2 and $4 \mathrm{~h}$. b Western blot analysis of HIF- $1 \alpha$ at protein level in HRMECs. Ad-Ctrl and $\mathrm{Ad}_{-} \mathrm{A}_{2 \mathrm{~A}} \mathrm{R}$-infected HRMECs were first treated with $\mathrm{CoCl} \mathrm{C}_{2}(200 \mu \mathrm{M})$ for $8 \mathrm{~h}$ to increase HIF-1 $\alpha$ protein level and then further treated with $\mathrm{CHx}(50 \mu \mathrm{M})$ for the indicated time periods. c, $\mathbf{d}$ The relative protein levels of HIF-1 $\alpha$ were quantified by comparing the intensities of protein bands at the indicated times to that at time $0 . n=3,{ }^{\star \star \star} P<0.001$. Data are represented as means \pm s.e.m. Statistical significance was determined by two-way ANOVA followed by Bonferroni test. e Western blot analysis of the levels of total-ERK ( $t$-ERK), phospho-ERK1/2 (p-ERK1/2), phospho-p38 (p-p38), total-p38 ( $t-p 38)$, phospho-JNK1/2 (p-JNK1/2), total-JNK1/2 ( $t-J N K 1 / 2)$, phospho-p70S6K $\left(p-p 70^{S 6 K}\right)$, total-p70 ${ }^{S 6 K}\left(t-p 70^{S 6 K}\right)$, phospho-elF-4E (p-elF-4E), and total-elF-4E (t-elF-4E) in HRMECs transfected with siA $A_{2 A} R$ or siCtrl, with or without adenosine treatment under hypoxia. $n=3$. $\mathbf{f}$ Western blot analysis of HIF- $1 \alpha$ and phosphoproteins in HRMECs infected with $A d-A_{2 A} R$, with or without adenosine treatment. $n=3$. $\mathbf{g}$ Western blot analysis of HIF-1 $\alpha$ and phosphoproteins in HRMECs infected with Ad-Ctrl and Ad- $A_{2 A} R$ in the presence of U0126 $(10 \mu \mathrm{M})$ or LY294002 $(10 \mu \mathrm{M}) . n=4$

vessel sprouting 8,10 , indicating that glycolysis is also critical for growth factor-driven angiogenesis. Interestingly, we show here that knockdown or deletion of endothelial ADORA2A dramatically inhibited gene expression of most glycolytic enzymes, including PFKFB3, in vitro and in vivo, resulting in decreased glycolysis under hypoxia conditions. However, it seems that ADORA2A knockdown or blockade has less effect on EC glycolysis under normoxia conditions. This may be due to the low levels of ADORA2A and adenosine in normoxic cells. In contrast, $A D O R A 2 A$ overexpression significantly upregulated glycolytic enzymes and increased glycolysis in the presence of adenosine. Blocking glycolysis with $3 \mathrm{PO}$ and 2-DG dramatically inhibited proliferation and hyper-sprouting of ADORA2A-overexpressing ECs. Overall, these loss- and gain-offunction assays strongly support that ADORA2A-regulated glycolysis is one of the critical pathways for ADORA2A-mediated angiogenesis.

HIFs are currently viewed as the major regulators of oxygen homeostasis, angiogenesis, and vascular permeability via induction of a host of pro-angiogenic genes and glycolytic enzymes $^{38}$. Proliferative retinopathies are characterized by hypoxia-induced pathological neovascularization driven by the HIF- $1 \alpha$ and HIF- $2 \alpha$ pathways ${ }^{39-41}$. HIF- $2 \alpha$ is a master regulator of proangiogenic factors in retinal vascular ECs. HIF- $2 \alpha$ haploinsufficiency results in a reduced ability to induce multiple proangiogenic factors and reduced neovascularization in the retinas of mice with $\mathrm{OIR}^{41}$. This study indicates HIF-2 $\alpha$ is able to upregulate ADORA2A. Likely, the angiogenic effects of HIF- $2 \alpha$ are, at least in part, mediated through ADORA2A-dependent signaling. Most of the glycolytic genes are identified as HIF- $1 \alpha$ 


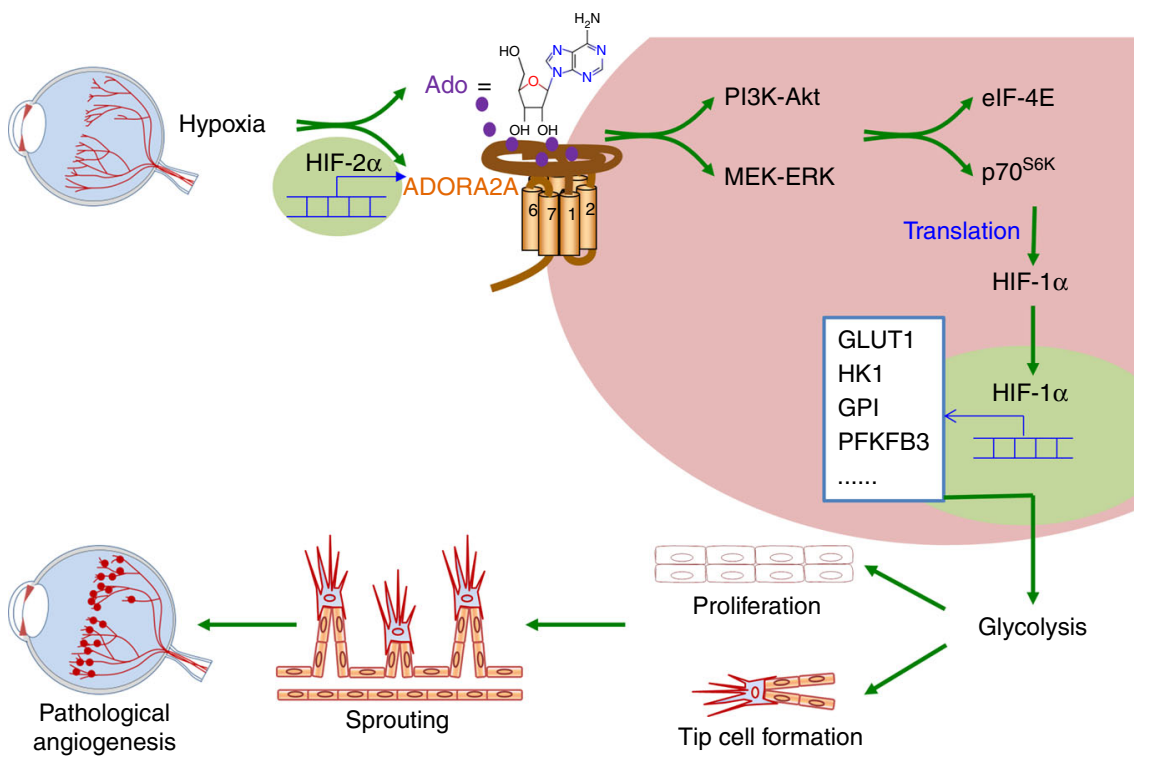

Fig. 10 Schematic diagram illustrating the molecular mechanisms underlying the angiogenic effect of adenosine-ADORA2A-mediated signaling cascade

target genes $25,26,42$. The expression of HIF-1 $\alpha$ can be modulated by different regulators through various mechanisms, including the basal transcription and translation machinery, as well as posttranscriptional and posttranslational mechanisms ${ }^{43}$. Ouyang et al. $^{44}$ showed that ADORA2A activation rapidly induced HIF- $1 \alpha$ mRNA expression via the cAMP/PKA/CREB pathway in macrophages. In contrast, $A D O R A 2 A$ knockdown or overexpression in retinal ECs did regulate the protein level of HIF-1 $\alpha$, but did not alter its mRNA level, implying that ADORA2A did not affect HIF-1 $\alpha$ transcription or mRNA stability. Our study further revealed that ADORA2A preferentially activates PI3K/Akt and MEK/ERK, two critical pathways responsible for HIF-1 $\alpha$ protein synthesis ${ }^{27}, 28,45$, as well as the downstream translational regulatory proteins of $\mathrm{p} 70^{\mathrm{S} 6 \mathrm{~K}}$ and eIF$4 \mathrm{E}$, without altering the phosphorylation of JNK1/2 and p38 MAPK kinase. Importantly, inhibitors of PI3K and MEK effectively blocked the ADORA2A activation-induced increase in HIF$1 \alpha$ protein, suggestive of a role for the PI3K and MEK-dependent translational machinery in ADORA2A-mediated upregulation of HIF- $1 \alpha$ protein levels. Additionally, the adenylyl cyclase inhibitor SQ22536 and PKA inhibitor H-89 did not affect translational induction of HIF-1 $\alpha$ (Supplementary Fig. 14), suggesting that the cAMP/PKA/CREB pathway is not involved in ADORA2Amediated HIF-1 $\alpha$ protein synthesis in HRMECs.

A salient observation of the present study is that ADORA2A knockdown impaired tip cell activity. Tip cells play a critical role in various models of sprouting angiogenesis, including proliferative retinopathy ${ }^{46}$. Tip cells are located at the forefront of vessel branches, and are highly polarized with numerous filopodia to probe the environment, and migrate toward angiogenic stimuli ${ }^{47,48}$. Recently a critical role of glycolysis in tip cell formation has been demonstrated ${ }^{8,9}$. Thus, a decrease in tip cell formation and tip cell activity for $A D O R A 2 A$ knockdown retinal ECs is, at least in part, dependent on ADORA2A-mediated glycolysis. Indeed, blockade of glycolysis by the glycolytic inhibitor 3PO and 2-DG markedly suppresses ADORA2A overexpression-induced endothelial sprouting. It has been shown that partial and transient reduction of glycolysis by PFKFB3 knockdown or blockade was not able to significantly alter the genetic tip or stalk cell signature of ECs ${ }^{8,9}$. Similarly, we found that short term treatment with the ADORA2A agonist CGS12680 or ATL313 did not affect transcript levels of Notch1, DLL4 or
Notch1 target genes HES1, HEY1, and HEY2 (Supplementary Fig. 15), indicating that ADORA2A activation probably does not directly regulate endothelial Notch signaling. However, we demonstrate here that systemic downregulation of glycolytic enzymes by $A D O R A 2 A$ knockdown or near-complete inhibition of glycolysis by 2-DG treatment downregulated tip-cell-enriched genes and upregulated stalk-cell-enriched genes. It is, therefore, likely that some of the glycolytic enzymes and/or their products regulate these genes. On the other hand, ADORA2A- or 2-DGregulated molecules include genes that broadly impact glucose metabolism rather than selectively suppress glycolysis. For example, both ADORA2A knockdown and 2-DG treatment can inhibit hexokinase-1 (HK1), an enzyme that phosphorylates glucose to produce glucose-6-phosphate (G6P), the first step in glucose metabolism. As such, many other pathways associated with glucose metabolism including reactive oxygen species and the pentose phosphate pathway (PPP) are affected ${ }^{7,49}$. This may contribute to the altered expression of tip-enriched genes. Additionally, it might be also possible that long-term or severe inhibition of glycolysis-associated signals by $A D O R A 2 A$ knockdown or 2-DG may eventually change endothelial homeostasis and consequently alter gene expression.

A recent genome-wide association study indicates a close association of vascular disease with the lead SNP in intronic regions of the $A D O R A 2 A$ gene ${ }^{50}$. In a recent study on PDR of patients with type 2 diabetes, microarray analysis of gene expression in fibrovascular membranes showed that the level of ADORA2A mRNA was much higher in samples excised from patients with PDR compared with those from patients with non-PDR ${ }^{51}$. In another PDR study, Charles et al. examined the associations between PDR and variants of the ADORA2A gene in a cohort of patients with type 1 diabetes. They found that among tagging SNPs (tSNPs; rs2236624-C/T, and rs4822489-G/T) in the ADORA2A gene, participants homozygous for the $\mathrm{T}$ allele displayed a decreased risk of developing prevalent $\mathrm{PDR}^{19}$. The data we collected from the GTEx database have shown that, compared with individuals homozygous for the $\mathrm{C}$ or $\mathrm{G}$ alleles, individuals homozygous for the $\mathrm{T}$ allele actually have a low level of ADORA2A mRNA in their blood cells (Supplementary Fig. 2), indicating that in Charles's study, the observation that type 1 diabetic patients have a low risk of developing PDR is very likely due to a low level of $A D O R A 2 A$ expression in their retinal ECs. 
With the results from this study showing that endothelial Adora2a knockdown or deletion reduces pathological retina angiogenesis, it may be safe to conclude that highly expressed ADORA2A, especially in retinal ECs, contributes to the development of pathological angiogenesis in the human retina.

Inhibiting pathological angiogenesis by targeting angiogenic factors such as VEGF has become an attractive clinical strategy in the treatment of proliferative retinopathies ${ }^{52}$. However, this approach has only achieved limited success. Recent advances in the suppression of retinal angiogenesis in preclinical studies have included therapies targeting EC glycolysis. Blocking the glycolytic regulator PFKFB3 suppresses retinal neovascularization in mouse models of OIR and AMD ${ }^{9,10}$. As shown in this study, ADORA2A is an upstream regulator for the HIF- $1 \alpha$-associated glycolytic pathway in retinal ECs, suggesting its dominant role in regulation of endothelial glycolysis and the associated angiogenic effect. Additionally, ADORA2A inhibition is also able to suppress VEGF-mediated angiogenesis. Hypoxia-mediated paracrine VEGF can induce EC expression of GLUT1 ${ }^{53}$ and PFKFB3 ${ }^{8}$ and subsequently promote glycolysis for vessel sprouting during ischemic retinopathies. Thus, the blocking of glycolysis by ADORA2A inactivation may also reduce the effect of VEGF on angiogenesis. Indeed, we found that $A D O R A 2 A$ knockdown led to a marked reduction in VEGF-induced HRMEC sprouting and tube formation, suggesting a facilitating role of ADORA2A in the VEGF pathway, the molecular target of current treatments for diabetic eye diseases. Over the past decade, ADORA2A antagonists have been generated to treat Parkinson's disease and have shown a good safety profile ${ }^{12}$. All these circumstances collectively indicate that targeting ADORA2A holds significant promise in the treatment of pathological angiogenesis in visionthreatening eye diseases and many other diseases highly dependent on pathological angiogenesis.

\section{Methods}

Chemicals and reagents. Recombinant hVEGF165 was from R\&D Systems (Minneapolis, MN, USA). Collagen type 1 (rat tail) was from BD Biosciences (Erembodegem, Belgium). Adenosine, erytho-9-(2-hydroxy-3-nonyl) adenine (EHNA), cobalt chloride $\left(\mathrm{CoCl}_{2}\right)$, and dimethyl sulfoxide were from Sigma-Aldrich (Bornem, Belgium). Calcein-AM, L-glutamine, and penicillin/streptomycin were from GIBCO (Grand Island, NY), and 4, 6,-diamidino-2-phenylindole (DAPI) was from Invitrogen (Invitrogen, Life Technologies, Ghent, Belgium). The PFKFB3 inhibitor 3-(3-pyridinyl)-1-(4-pyridinyl)-2-propen-1-one (3PO), the glycolysis inhibitor 2-deoxy-D-glucose (2-DG), and the $\gamma$-secretase inhibitor $\mathrm{N}$-[N-(3,5-Difluorophenacetyl)-L-alanyl]-S-phenylglycine t-butyl ester (DAPT) were from Merck Millipore (Overijse, Belgium).

\begin{abstract}
Mouse generation and breeding. Animals were used according to the National Institutes of Health Guide for the Care and Use of Laboratory Animals and in accordance with the protocol approved by the Institutional Animal Care and Use Committee at the Augusta University. The floxed Adora2a (Adora2a flox/flox $)$ mice were provided by Dr Joel Linden (La Jolla Institute for Allergy and Immunology, La Jolla, California, USA). Cell-specific inactivation of Adora2a in ECs or in macrophages was achieved by cross-breeding Adora2 $a^{\text {flox/flox }}$ mice with Cdh5-Cre transgenic mice (The Jackson Laboratory, Stk\#006137, Bar Harbor, ME) or Lysm-cre transgenic mice (The Jackson Laboratory, Stk\#004781), respectively.

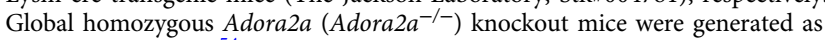
previously described ${ }^{54}$. All mice were on a C57BL/6J background.
\end{abstract}

Mouse model of OIR. The OIR model was described previously ${ }^{55}$. Briefly, sevenday-old (P7) C57BL/6J mouse pups, including both males and females, along with the foster/nursing mothers, were exposed to $70 \%$ or $75 \% \mathrm{O}_{2}$ for 5 days to induce vaso-obliteration. At P12, the mice were returned to room air (RA, $\left.21 \% \mathrm{O}_{2}\right)$ to induce retinal neovascularization, which was maximal at P17. Age-matched mice kept in RA throughout postnatal development (P0-P17) served as the RA controls. Underdeveloped neonatal mice with weight less than $6 \mathrm{~g}$ at P17 were excluded.

Laser-capture microdissection of retinal vessels. Retinal vessels were microdissected with laser capture in retinal cross sections from Adora $2 a^{\text {flox/flox }} C \mathrm{dh} 5^{\text {cre }}$ $\left(\right.$ Adora $\left.2 a^{V E C-K O}\right)$ and Adora2a flox/flox $\left(\right.$ Adora2a $\left.a^{W T}\right)$ mice at postnatal day $(\mathrm{P}) 17$, as described previously ${ }^{2}, 56$. In brief, eyes were embedded in OCT and flash frozen immediately following enucleation. Eyes were cyrosectioned under RNase free conditions into $10-\mu \mathrm{m}$ sections, and collected on RNase-free polyethylene naphthalate glass slides (11505189, Leica). Sections were dehydrated with 70, 90, and $100 \%$ ethanol washes and stained with isolectin $\left(1: 50\right.$ in $\left.1 \mathrm{mM} \mathrm{CaCl}_{2}\right)$. Retinal vessels were microdissected with a Leica LMD 6000 system (Leica Microsystems) and collected directly into RNA stabilizing buffer from the RNeasy Micro kit (Qiagen, Chatsworth, CA). RNA was extracted from microdissected tissues using the RNeasy kit as described above (Qiagen), and Real-Time PCR was performed with the generated cDNA.

Isolation of mouse retinal endothelial cells. Isolation of MAECs was performed according to protocols as described previously with some modifications $s^{57}$. Briefly, eyes from one litter (5 to 6 pups) of OIR-Adora $2 a^{V E C-K O}$ and Adora $2 a^{W T}$ mice at P17 were enucleated and hemisected. The retinas were dissected out and kept in pre-cooling phosphate-buffered saline (PBS) buffer. Retinas (10 to 12 from one litter) were pooled together, rinsed with PBS buffer, quickly minced into small pieces in a $1.5 \mathrm{ml}$ tube using eye scissors, and digested in $8 \mathrm{ml}$ of collagenase type II (Worthington, $2 \mathrm{mg} / \mathrm{ml}$ in serum free DMEM, Corning, NY, USA) for $20 \mathrm{~min}$ at $37^{\circ} \mathrm{C}$. Following digestion, DMEM with $20 \%$ FBS was added and cells were pelleted. The cellular digests then were filtered through $70-\mu \mathrm{m}$ and $40-\mu \mathrm{m}$ nylon filters (Corning, NY, USA), centrifuged at $500 \times g$ for $5 \mathrm{~min}$ at $4{ }^{\circ} \mathrm{C}$ to pellet cells, and cells were washed with pre-cooling PBS containing $0.5 \%$ bovine serum albumin (BSA). The cells were resuspended in $100 \mu \mathrm{l}$ pre-cooled PBS containing $0.5 \%$ BSA and $2 \mathrm{mM}$ EDTA, and incubated with CD31-MicroBeads for $10 \mathrm{~min}$ at $4^{\circ} \mathrm{C}$ (Miltenyi Biotec Inc). After affinity binding, CD31-positive cells were obtained via magnetic separation using a MACS separator (Miltenyi Biotec). Purified ECs were immediately lysed for Real-Time PCR assay.

Cell culture and treatments. Human primary retinal microvascular ECs (HRMECs) were obtained from Cell Biologics (Cat. No. H-6065; Chicago, IL, USA) and used between passages 3-8. The type of cells and no pathogen (including mycoplasma) contamination were confirmed by the supplier.

HRMECs were cultured in Vessel Cell Basal Medium (VCBM, ATCC Manassas, VA, USA) supplemented with Microvascular Endothelial Cell Growth Kit-BBE (ATCC), and 1\% penicillin/streptomycin, or in Complete Human Endothelial Cell Medium (Cell Biologics). In some experiments, HRMECs were incubated with $20 \mathrm{ng} / \mathrm{ml}$ recombinant hVEGF, $10 \mu \mathrm{M}$ DAPT, $200 \mu \mathrm{M} \mathrm{CoCl}_{2}$ or 20-100 $\mu \mathrm{M}$ adenosine in the presence of $10 \mu \mathrm{M}$ EHNA according to the protocol previously described ${ }^{58,59}$. For the experiments requiring hypoxia, HRMECs were placed in a modular incubator chamber (Thermo Scientific, Waltham, MA) with $0.1-2 \% \mathrm{O}_{2}$

Adenoviral transduction of HRMECs. Ad- $\mathrm{A}_{2 \mathrm{~A}} \mathrm{R}$, a recombinant adenovirus vector expressing human $A D O R A 2 A$; Ad-mutHIF- $1 \alpha$ encoding the mutant human HIF- $1 \alpha$ construct containing mutations at P564A and N803A; and Ad-mutHIF- $2 \alpha$ containing mutations at P531A and N847A, were generated as previously described ${ }^{60}$. Expressed mutHIF- $1 \alpha$ and mutHIF- $2 \alpha$ are stable and constitutively active under normoxic conditions ${ }^{60}$. Adenovirus encoding the inert Escherichia coli LacZ gene was used as a negative control (Ad-Ctrl). These adenoviruses were expanded in HEK293 cells, and the virus concentration was determined using an Adeno-XTM rapid titer kit (Clontech). Adenoviral infection of HRMECs were carried out at a multiplicity of infection of 10 pfu per cell, as described previously ${ }^{60}$

RNA interference. HRMECs were transfected at $60-70 \%$ confluence with $30 \mathrm{nM}$ siRNAs targeting human ADORA2A (Adenosine A2A-R siRNA ( siA $_{2 \mathrm{~A}} \mathrm{R}$ ), Cat. No. sc-39850; Santa Cruz Biotechnology, Dallas, Texas, USA) or with a non-targeting negative control (Control siRNA-A (siCtrl), Cat. No. sc-37007; Santa Cruz Biotechnology) using siRNA transfection reagent (Santa Cruz Biotechnology) or Lipofectamine RNAiMAX Reagent (Invitrogen) per the manufacturer's protocol. Knockdown of HIF- $1 \alpha$ and HIF- $2 \alpha$ in HRMECs was carried out by using predesigned SmartPool siRNA purchased from Dharmacon (siHIF-1 $\alpha$, Cat. No. L-004018; siHIF-2 $\alpha$, Cat. No. L-004814; Lafayette, CO). Twenty-four hours after transfection, the medium was changed to fresh complete VCBM, and cells were maintained for an additional $24 \mathrm{~h}$ before further experiments.

Spheroid capillary sprouting assay. HRMECs (750 cells) were incubated overnight in 25\% VCBM (25\% VCBM complete medium + 75\% VCBM basal medium) containing $0.25 \%(\mathrm{w} / \mathrm{v})$ methylcellulose (Sigma-Aldrich) to form spheroids as described previously ${ }^{61,62}$. To assess tip cell competition, cells were mixed at a 1:1 ratio. After $24 \mathrm{~h}$, spheroids were harvested and embedded in $0.9 \mathrm{ml}$ collagen solution in pre-warmed 24-well plates, with a final concentration of rat type I collagen (BD Biosciences) at $1.5 \mathrm{mg} / \mathrm{ml}$. The spheroid-containing gels were rapidly transferred into a humidified incubator $\left(37^{\circ} \mathrm{C}, 5 \% \mathrm{CO}_{2}\right)$ and allowed to polymerize $(20 \mathrm{~min})$ after which $0.1 \mathrm{ml}$ VCBM basal medium was pipetted on top of the gel containing the corresponding cytokines or compounds. After $24 \mathrm{~h}$, cells were fixed with pre-warmed $4 \%$ paraformaldehyde (PFA), stained with DRAQ5 (Thermo Scientific) to mark EC nuclei and imaged using a Zeiss LSM 780 Inverted Confocal Microscope. The number of sprouts and cumulative length of sprouts per spheroid were quantified from 10 spheroids for each condition using Image J software. 
Fluorescence immunostaining in whole-mount retinas. OIR-mice at P17 were euthanized and perfused successively with PBS and 4\% PFA, and the intact retinas were collected. Retinas were blocked and permeabilized in PBS containing $10 \%$ goat serum and 1\% Triton-X-100 (Sigma-Aldrich) for 30 min. Endogenous Fc receptors and IgG were blocked with rat anti-mouse CD16/CD32 (Mouse BD Block $^{\mathrm{TM}}, 1: 50$, BD Biosciences, 553142) and the blocking reagent provided in the mouse-on-mouse kit (Vector Laboratories, Cat. No. FMK-2201, Burlingame, CA, USA), respectively. Retinas were then incubated with primary antibodies against mouse Adora2a (1:100, Millipore, 05-717), rabbit IBa1 (1:400, Sakura Finetek, 019-19741, Torrance, CA), and Alexa488- or Alexa-594 labeled Griffonia simplicifolia isolectin B4 (1:200, Invitrogen, 121411 and 121413, Carlsbad, CA, USA) overnight at $4{ }^{\circ} \mathrm{C}$, followed by incubation with fluorescence-conjugated cross-adsorbed secondary antibody (1:500, Molecular Probes, Life Technologies, A-21131, Carlsbad, CA,USA) for 1 hour, and then counterstained with DAPI (Invitrogen). Retinas were flat mounted on microscope slides in mounting medium (Vectashield; Vector Laboratories) and examined by confocal microscopy (Zeiss 780; Carl Zeiss, Jena, Germany). Areas of vaso-obliteration and vitreoretinal neovascular tufts were quantified using Adobe Photoshop CS 5 software.

Immunofluorescence of eye sections. Eyes were fixed in $4 \%$ PFA for $2 \mathrm{~h}$ at room temperature and equilibrated in $30 \%$ sucrose at $4{ }^{\circ} \mathrm{C}$, followed by embedding in OCT. Sections $\left(10-\mu \mathrm{m}\right.$ thick) were heated at $98^{\circ} \mathrm{C}$ for $10 \mathrm{~min}$ in citric acid buffer for antigen retrieval, blocked with $10 \%$ goat serum for 1 hour, and incubated with mouse HIF-1 $\alpha$ (1:100, BD Biosciences, 610958), rabbit Ki-67 (1:200, RM-9106, Thermo Scientific), rabbit PFKFB3 (1:100, Proteintech, 13763-1-AP), rat CD31 (1:25, Invitrogen, DIA-310) and/ or Alexa-594 labeled Griffonia simplicifolia isolectin B4 (1:100, Invitrogen, Cat. No. 121413) overnight at $4{ }^{\circ} \mathrm{C}$, followed by incubation with fluorescence-conjugated secondary antibody (1:250, Molecular Probes, Life Technologies, Carlsbad, CA,USA) for 1 hour. For Ki-67/ ERG double immunofluorescent staining, sections were then stained with Anti-ERG antibody (Alexa Fluor $\left.{ }^{\circledR} 594\right)\left(1: 200\right.$, Abcam, Clone number: EPR3864) overnight at $4{ }^{\circ} \mathrm{C}$. Sections were washed with PBS, immersed in ProLong Gold mounting medium with DAPI (Invitrogen) to visualize the nuclei, and examined using confocal microscopy. For all immunofluorescence experiments, parallel groups of sections were stained with only primary or secondary antibody as negative controls.

Neovascular nuclei quantification. To quantify neovascular nuclei, retina sections of OIR mice were stained with hematoxylin-eosin (H\&E). The extent of neovascularization was evaluated by counting the number of neovascular nuclei, which were defined as the nuclei of cells that extended beyond the inner limiting membrane of the retina into the vitreous. In this study, eyes of 6-8 mice from each group were examined and analyzed. Neovascular nuclei were counted in cross-sections with light microscopy under $40 \times$ magnification by an investigator who was blinded to the specific group assignment.

Real-Time PCR analysis. Total RNA of HRMECs and retinas were extracted using Trizol Reagent (Invitrogen, Grand Island, NY). In all, 0.1-1.0 $\mu \mathrm{g}$ sample of total RNA was utilized as a template for reverse transcription using the QuantiTect Reverse Transcription Kit (QIAGEN) for HRMECs or iScriptTM cDNA synthesis kit (Bio-Rad) for retinas. Real-time PCR was performed on a StepOne Plus System (Applied Biosystems, Grand Island, NY) using Power SYBR GreenMaster Mix (Applied Biosystems) with the respective gene-specific primers listed in Supplementary Table 1. Quantification of relative gene expression was calculated with the efficiency-corrected $2^{-} \triangle \triangle \mathrm{CT}$ method using GUBS and 18S rRNA (for human RNA), or HMBS and HRPT (for mouse RNA) as the internal control, and data were presented as fold change relative to control groups.

Protein extraction and western blot. HRMECs were lysed with RIPA buffer (Fisher) supplemented with $1 \%$ proteinase inhibitor cocktail (Pierce, Rockford, IL) and $1 \%$ phosphatase inhibitors (Pierce). After sonication and centrifugation of cell lysates, protein was quantified with the BCA assay and then loaded in the $8 \%$ sodium dodecyl sulfate polyacrylamide gel electrophoresis (SDS-PAGE) gel at $10-20 \mu \mathrm{g}$ per lane. Primary antibodies used in this study were as follows: ADORA2A (Sigma, A-269; rabbit, 1:500), ADORA2A (Millipore, 05-717; Mouse, 1:1000), HIF-1 $\alpha$ (BD Biosciences, 610958; mouse, 1:500), HIF-1 $\alpha$ (R\&D Systems, AF1935; goat, 1:1000), HIF-2 $\alpha$ (Novus Biologicals, NB100-122; Littleton, CO, USA; rabbit, 1:1000), PFKFB3 (Proteintech, 13763-1-AP; rabbit, 1:2000), p-AKT (Cell Signaling Technology, 4060; rabbit, 1:2000), AKT (Cell Signaling Technology, 4691; rabbit, 1:2000), p-ERK1/2 (Cell Signaling Technology, 4370; rabbit, 1:2000), ERK1/2 (Cell Signaling Technology, 4695; rabbit, 1:2000), p-p38 (Cell Signaling Technology, 9215; rabbit, 1:1000), p38 (Cell Signaling Technology, 8690; rabbit, 1:1000), p-JNK1/2 (Cell Signaling Technology, 9251; rabbit, 1:1000), JNK1/2 (Cell Signaling Technology, 9252; rabbit, 1:1000), p-p70 ${ }^{\mathrm{S} 6 \mathrm{~K}}$ (Cell Signaling Technology, 9234; rabbit, 1:1000), p70 ${ }^{\text {S6K }}$ (Cell Signaling Technology, 2708; rabbit, 1:1000), p-eIF-4E (Cell Signaling Technology, 9741; rabbit, 1:1000), eIF-4E (Cell Signaling Technology, 2067; rabbit, 1:1000), and $\beta$-actin (Cell Signaling Technology, 3700; mouse, 1:5000). Images were taken with the ChemiDoc MP system (Bio-Rad), and band densities were quantified using Image Lab software (Bio-Rad). Uncropped scans for western blots are provided in Supplementary Figs. 16-18.
Capillary tube network formation. HRMECs were seeded on growth factorreduced Matrigel (BD Bioscience)-coated 96-well plates $\left(1 \times 10^{4}\right.$ cells per well) in $0.1 \mathrm{ml} \mathrm{VCBM} \mathrm{for} 4 \mathrm{~h}$. The endothelial tubule formation was observed and photographed using an inverted confocal microscope after staining with Calcein AM. Cumulative tube length was quantified using the Image J software. Branch points were manually counted.

Metabolic measurements. HRMECs were seeded on Seahorse XF96 polystyrene tissue culture plates (Seahorse Bioscience, North Billerica, MA), and incubated at $37^{\circ} \mathrm{C}$ overnight in $25 \%$ VCBM. To avoid differences due to unequal cell numbers and growth rates, all measurements were made starting with confluent cells by seeding $1.5 \times 10^{4}$ per well. The next day, the medium was changed to XF base Medium (Seahorse Bioscience) supplemented with $2 \mathrm{mM}$ glutamine (for ECAR), or supplemented with $25 \mathrm{mM}$ glucose, $1 \mathrm{mM}$ pyruvate, and $2 \mathrm{mM}$ glutamine (for oxygen consumption rate, OCR), and then the plate was incubated for $1 \mathrm{~h}$ in a non- $\mathrm{CO}_{2}$ incubator at $37^{\circ} \mathrm{C}$. ECAR and OCR were measured with an $\mathrm{XF}^{\mathrm{e}} 96$ extracellular flux analyzer (Seahorse Bioscience). Inhibitors and activators were used in these tests at the following concentrations: glucose $(10 \mathrm{mM})$, oligomycin $(2 \mu \mathrm{M}), 2$-DG $(50 \mathrm{mM})$, FCCP $(1 \mu \mathrm{M})$, antimycin A $(0.5 \mu \mathrm{M})$, and rotenone $(0.5 \mu \mathrm{M})$

Lactate measurements. The levels of secreted lactate in cell medium of HRMECs were determined using the Lactate Assay Kit (Sigma-Aldrich, Cat. No. MAK064)

WST-1 proliferation assay. HRMECs were seeded at $4 \times 10^{3}$ cells per well in 96-well plates. The cells were incubated in 25\% VCBM for $72 \mathrm{~h}$ under normoxia $\left(21 \% \mathrm{O}_{2}\right)$ or hypoxia $\left(0.5 \% \mathrm{O}_{2}\right)$ and proliferation was assessed by WST-1 assay (Sigma-Aldrich, Cat. No. 5015944001).

Ki-67 staining and BrdU incorporation analysis. HRMECs were treated with $\mathrm{BrdU}$ labeling reagent (Invitrogen) for $16 \mathrm{~h}$. Following BrdU treatment, cells were fixed with 4\% PFA for $10 \mathrm{~min}$, permeabilized in PBS containing $0.5 \%$ Triton-X-100 for $15 \mathrm{~min}$, treated with $2 \mathrm{~N} \mathrm{HCl}$ for $30 \mathrm{~min}$, blocked with $10 \%$ goat serum for $1 \mathrm{~h}$ and then incubated with a mouse monoclonal anti-BrdU antibody (1:200, Invitrogen, Cat. No. 03-3900) and rabbit anti-Ki-67 antibody (1:200, Thermo Scientific, Cat. No. RM-9106) overnight at $4{ }^{\circ} \mathrm{C}$, followed by incubation with fluorescenceconjugated secondary antibody (1:250, Molecular Probes, Life Technologies, Carlsbad, CA, USA) for $1 \mathrm{~h}$. The cells were then immersed in ProLong Gold mounting medium with DAPI (Invitrogen) to visualize the nuclei. Images were obtained using an inverted fluorescence microscope (Zeiss Axio Observer Z1) or upright confocal microscope (Zeiss 780; Carl Zeiss). The number of Ki-67 or BrdU-positive cells was counted in six non-overlapping and randomly selected microscopic fields per slide.

Statistical analysis. The optimal animal numbers and sample sizes were estimated based on power analysis, prior experience, and our preliminary data. Grouping was performed in a randomized manner when using C57BL/6J wild-type mice. No randomization was used when using other mice, since all these mice were genetically defined, inbred mice. Data analysis for in vivo angiogenic phenotype was performed in a blinded fashion. Data are presented as means \pm s.e.m. Statistical analysis was performed using GraphPad Prism Software (La Jolla, CA). After the normal distribution was confirmed with the Kolmogorov-Smirnov test, statistical comparisons were done using two-tailed unpaired Student's $t$-test or one- or twoway analysis of variance (ANOVA) followed by Bonferroni's post hoc tests when appropriate. Two-sided $P$-values were calculated and $P<0.05$ denoted significance. Statistical significance was defined as follows: ${ }^{*} P<0.05,{ }^{* *} P<0.01,{ }^{* * *} P<0.001$.

Data availability. The authors state that all relevant data are available within the article and its Supplementary Information files or are available from the corresponding authors upon reasonable request.

Received: 17 November 2016 Accepted: 21 June 2017

Published online: 19 September 2017

\section{References}

1. Penn, J. S. et al. Vascular endothelial growth factor in eye disease. Prog. Retin. Eye. Res. 27, 331-371 (2008).

2. Chen, J. et al. Wnt signaling mediates pathological vascular growth in proliferative retinopathy. Circulation 124, 1871-1881 (2011).

3. Rama, N. et al. Slit2 signaling through Robo1 and Robo2 is required for retinal neovascularization. Nat. Med. 21, 483-491 (2015).

4. Horowitz, A. \& Simons, M. Branching morphogenesis. Circ. Res. 103, 784-795 (2008).

5. Carmeliet, P. \& Tessier-Lavigne, M. Common mechanisms of nerve and blood vessel wiring. Nature 436, 193-200 (2005). 
6. Doddaballapur, A. et al. Laminar shear stress inhibits endothelial cell metabolism via KLF2-mediated repression of PFKFB3. Arterioscler. Thromb. Vasc. Biol. 35, 137-145 (2015)

7. Eelen, G., de Zeeuw, P., Simons, M. \& Carmeliet, P. Endothelial cell metabolism in normal and diseased vasculature. Circ. Res. 116, 1231-1244 (2015).

8. De Bock, K. et al. Role of PFKFB3-driven glycolysis in vessel sprouting. Cell 154, 651-663 (2013).

9. Schoors, S. et al. Partial and transient reduction of glycolysis by PFKFB3 blockade reduces pathological angiogenesis. Cell Metab. 19, 37-48 (2014).

10. $\mathrm{Xu}, \mathrm{Y}$. et al. Endothelial PFKFB3 plays a critical role in angiogenesis. Arterioscler. Thromb. Vasc. Biol. 34, 1231-1239 (2014).

11. Barba, I. et al. Metabolic fingerprints of proliferative diabetic retinopathy: an $1 \mathrm{H}-\mathrm{NMR}$-based metabonomic approach using vitreous humor. Invest. Ophthalmol. Vis. Sci. 51, 4416-4421 (2010).

12. Chen, J. F., Eltzschig, H. K. \& Fredholm, B. B. Adenosine receptors as drug targets--what are the challenges? Nat. Rev. Drug Discov. 12, 265-286 (2013).

13. Lutty, G. A., Merges, C. \& McLeod, D. S. 5' nucleotidase and adenosine during retinal vasculogenesis and oxygen-induced retinopathy. Invest. Ophthalmol. Vis. Sci. 41, 218-229 (2000).

14. Lutty, G. A. \& McLeod, D. S. Retinal vascular development and oxygen-induced retinopathy: a role for adenosine. Prog. Retin. Eye. Res. 22, 95-111 (2003).

15. Montesinos, M. C., Shaw, J. P., Yee, H., Shamamian, P. \& Cronstein, B. N Adenosine $\mathrm{A}(2 \mathrm{~A})$ receptor activation promotes wound neovascularization by stimulating angiogenesis and vasculogenesis. Am. J. Pathol. 164, 1887-1892 (2004).

16. Montesinos, M. C. et al. Adenosine promotes wound healing and mediates angiogenesis in response to tissue injury via occupancy of $\mathrm{A}(2 \mathrm{~A})$ receptors. Am. J. Pathol. 160, 2009-2018 (2002).

17. Bogenpohl, J. W., Ritter, S. L., Hall, R. A. \& Smith, Y. Adenosine A2A receptor in the monkey basal ganglia: ultrastructural localization and colocalization with the metabotropic glutamate receptor 5 in the striatum. J. Comp. Neurol. 520, 570-589 (2012)

18. Lazarus, M. et al. Arousal effect of caffeine depends on adenosine A2A receptors in the shell of the nucleus accumbens. J. Neurosci. 31, 10067-10075 (2011).

19. Charles, B. A. et al. Variants of the adenosine A(2A) receptor gene are protective against proliferative diabetic retinopathy in patients with type 1 diabetes. Ophthalmic. Res. 46, 1-8 (2011).

20. Birdsey, G. M. et al. The endothelial transcription factor ERG promotes vascular stability and growth through $\mathrm{Wnt} /$ beta-catenin signaling. Dev. Cell 32, 82-96 (2015).

21. Choi, H. J. et al. Yes-associated protein regulates endothelial cell contactmediated expression of angiopoietin-2. Nat. Commun. 6, 6943 (2015).

22. De Bock, K., Georgiadou, M. \& Carmeliet, P. Role of endothelial cell metabolism in vessel sprouting. Cell Metab. 18, 634-647 (2013)

23. Potente, M., Gerhardt, H. \& Carmeliet, P. Basic and therapeutic aspects of angiogenesis. Cell 146, 873-887 (2011).

24. Hellstrom, M. et al. Dll4 signalling through Notch1 regulates formation of tip cells during angiogenesis. Nature 445, 776-780 (2007).

25. Iyer, N. V. et al. Cellular and developmental control of $\mathrm{O} 2$ homeostasis by hypoxia-inducible factor 1 alpha. Genes Dev. 12, 149-162 (1998).

26. Wenger, R. H. Mammalian oxygen sensing, signalling and gene regulation. J. Exp. Biol. 203, 1253-1263 (2000).

27. Fukuda, R. et al. Insulin-like growth factor 1 induces hypoxia-inducible factor 1-mediated vascular endothelial growth factor expression, which is dependent on MAP kinase and phosphatidylinositol 3-kinase signaling in colon cancer cells. J. Biol. Chem. 277, 38205-38211 (2002).

28. Choi, Y. K. et al. Carbon monoxide promotes VEGF expression by increasing HIF-1alpha protein level via two distinct mechanisms, translational activation and stabilization of HIF-1alpha protein. J. Biol. Chem. 285, 32116-32125 (2010).

29. Semenza, G. L. Targeting HIF-1 for cancer therapy. Nat. Rev. Cancer 3, 721-732 (2003)

30. Liu, X. L. et al. Genetic inactivation of the adenosine A2A receptor attenuates pathologic but not developmental angiogenesis in the mouse retina. Invest. Ophthalmol. Vis. Sci. 51, 6625-6632 (2010).

31. Ohno-Matsui, K. et al. Inducible expression of vascular endothelial growth factor in adult mice causes severe proliferative retinopathy and retinal detachment. Am. J. Pathol. 160, 711-719 (2002).

32. Kollias, A. N. \& Ulbig, M. W. Diabetic retinopathy: early diagnosis and effective treatment. Dtsch. Arztebl. Int. 107, 75-83 (2010).

33. Lee, $\mathrm{S}$. et al. Autocrine VEGF signaling is required for vascular homeostasis. Cell 130, 691-703 (2007).

34. Leibovich, S. J. et al. Synergistic up-regulation of vascular endothelial growth factor expression in murine macrophages by adenosine $\mathrm{A}(2 \mathrm{~A})$ receptor agonists and endotoxin. Am. J. Pathol. 160, 2231-2244 (2002).
35. Vander Heiden, M. G., Cantley, L. C. \& Thompson, C. B. Understanding the Warburg effect: the metabolic requirements of cell proliferation. Science 324, 1029-1033 (2009).

36. Vander Heiden, M. G. et al. Evidence for an alternative glycolytic pathway in rapidly proliferating cells. Science 329, 1492-1499 (2010).

37. Locasale, J. W. \& Cantley, L. C. Altered metabolism in cancer. BMC Biol. 8, 88 (2010).

38. Krock, B. L., Skuli, N. \& Simon, M. C. Hypoxia-induced angiogenesis: good and evil. Genes Cancer 2, 1117-1133 (2011)

39. Xia, X. B., Xiong, S. Q., Xu, H. Z., Jiang, J. \& Li, Y. Suppression of retinal neovascularization by shRNA targeting HIF-1alpha. Curr. Eye Res. 33, 892-902 (2008).

40. Jiang, J. et al. Inhibition of retinal neovascularization by gene transfer of small interfering RNA targeting HIF-1alpha and VEGF. J. Cell. Physiol. 218, 66-74 (2009).

41. Dioum, E. M., Clarke, S. L., Ding, K., Repa, J. J. \& Garcia, J. A. HIF-2alphahaploinsufficient mice have blunted retinal neovascularization due to impaired expression of a proangiogenic gene battery. Invest. Ophthalmol. Vis. Sci. 49, 2714-2720 (2008).

42. Rowe, I. et al. Defective glucose metabolism in polycystic kidney disease identifies a new therapeutic strategy. Nat. Med. 19, 488-493 (2013).

43. Semenza, G. L. Hypoxia-inducible factor 1 (HIF-1) pathway. Sci. STKE 2007, cm8 (2007)

44. Ouyang, X. et al. Adenosine is required for sustained inflammasome activation via the $\mathrm{A}(2) \mathrm{A}$ receptor and the HIF-1alpha pathway. Nat. Commun. 4, 2909 (2013).

45. Laughner, E., Taghavi, P., Chiles, K., Mahon, P. C. \& Semenza, G. L. HER2 (neu) signaling increases the rate of hypoxia-inducible factor 1alpha (HIF-1alpha) synthesis: novel mechanism for HIF-1-mediated vascular endothelial growth factor expression. Mol. Cell. Biol. 21, 3995-4004 (2001).

46. Siemerink, M. J., Klaassen, I., Van Noorden, C. J. \& Schlingemann, R. O. Endothelial tip cells in ocular angiogenesis: potential target for antiangiogenesis therapy. J. Histochem. Cytochem. 61, 101-115 (2013).

47. Gerhardt, H. et al. VEGF guides angiogenic sprouting utilizing endothelial tip cell filopodia. J. Cell. Biol. 161, 1163-1177 (2003).

48. Ruhrberg, C. et al. Spatially restricted patterning cues provided by heparinbinding VEGF-A control blood vessel branching morphogenesis. Genes Dev. 16, 2684-2698 (2002).

49. Ghesquiere, B., Wong, B. W., Kuchnio, A. \& Carmeliet, P. Metabolism of stromal and immune cells in health and disease. Nature 511, 167-176 (2014).

50. Nikpay, M. et al. A comprehensive 1000 Genomes-based genome-wide association meta-analysis of coronary artery disease. Nat. Genet. 47, 1121-1130 (2015).

51. Ishikawa, K. et al. Microarray analysis of gene expression in fibrovascular membranes excised from patients with proliferative diabetic retinopathy. Invest Ophthalmol. Vis. Sci. 56, 932-946 (2015).

52. Singh, M. \& Ferrara, N. Modeling and predicting clinical efficacy for drugs targeting the tumor milieu. Nat. Biotechnol. 30, 648-657 (2012).

53. Yeh, W. L., Lin, C. J. \& Fu, W. M. Enhancement of glucose transporter expression of brain endothelial cells by vascular endothelial growth factor derived from glioma exposed to hypoxia. Mol. Pharmacol. 73, 170-177 (2008).

54. Chen, J. F. et al. A(2A) adenosine receptor deficiency attenuates brain injury induced by transient focal ischemia in mice. J. Neurosci. 19, 9192-9200 (1999).

55. Connor, K. M. et al. Quantification of oxygen-induced retinopathy in the mouse: a model of vessel loss, vessel regrowth and pathological angiogenesis. Nat. Protoc. 4, 1565-1573 (2009).

56. Joyal, J. S. et al. Retinal lipid and glucose metabolism dictates angiogenesis through the lipid sensor Ffar1. Nat. Med. 22, 439-445 (2016).

57. Su, X., Sorenson, C. M. \& Sheibani, N. Isolation and characterization of murine retinal endothelial cells. Mol. Vis. 9, 171-178 (2003).

58. Gessi, S. et al. Adenosine modulates HIF-1\{alpha\}, VEGF, IL-8, and foam cell formation in a human model of hypoxic foam cells. Arterioscler. Thromb. Vasc Biol. 30, 90-97 (2010).

59. Zhang, Y., Handy, D. E. \& Loscalzo, J. Adenosine-dependent induction of glutathione peroxidase 1 in human primary endothelial cells and protection against oxidative stress. Circ. Res. 96, 831-837 (2005).

60. Ahmad, A. et al. Adenosine A2A receptor is a unique angiogenic target of HIF-2alpha in pulmonary endothelial cells. Proc. Natl Acad. Sci. USA 106, 10684-10689 (2009).

61. Korff, T. \& Augustin, H. G. Integration of endothelial cells in multicellular spheroids prevents apoptosis and induces differentiation. J. Cell. Biol. 143, 1341-1352 (1998).

62. Heiss, M. et al. Endothelial cell spheroids as a versatile tool to study angiogenesis in vitro. FASEB J. 29, 3076-3084 (2015) 


\section{Acknowledgements}

We appreciate Yan V. Sun (Department of Epidemiology, Rollins School of Public Health, Emory University), and Hongyan Xu (Department of Biostatistics and Epidemiology, Medical College of Georgia, Augusta University) for collecting data from http://www.gtexportal.org/home. This work was supported by grants from National Key Basic Research Program of China (2012CB910402), National Natural Science Foundation of China (81400826), Guangdong Natural Science Foundation (2014A030312004), the Shenzhen Science and Technology Innovation Committee (20160517084712652, 20160503001803075, JCYJ20140903101709818,

JSGG20140717102922014), the Shenzhen Peacock Program (KQCX2015032709315529), American Heart Association (16GRNT30510010), and the National Institutes of Health (HL095556, R01DK095862)

\section{Author contributions}

Z.L., R.B.C., J.-F.C., and Y.H. designed research; Z.L., S.Y., J.W., Y.X., Y.W., S.Z., X.X., Q.Y., X.Z., Y.Z., X.G., I.K.-D., A.S.I., and Z.F. performed experiments; Z.L., S.Y., J.W., I.K.-D., M.A.-S., S.L., and Y.H. analyzed data; Z.L., D.J.F., N.L.W., W.Z., M.A.-S., A.S., and Y.H. wrote or revised the manuscript; Q.L., A.A., Y.S., X.J., X.-L.L., C.W., A.S., R.B. C., L.S., M.H., and Y.H. provided the reagents or materials and participated in discussing experiments.

\section{Additional information}

Supplementary Information accompanies this paper at doi:10.1038/s41467-017-00551-2.
Competing interests: The authors declare no competing financial interests.

Reprints and permission information is available online at http://npg.nature.com/ reprintsandpermissions/

Publisher's note: Springer Nature remains neutral with regard to jurisdictional claims in published maps and institutional affiliations.

(c) (i) Open Access This article is licensed under a Creative Commons Attribution 4.0 International License, which permits use, sharing, adaptation, distribution and reproduction in any medium or format, as long as you give appropriate credit to the original author(s) and the source, provide a link to the Creative Commons license, and indicate if changes were made. The images or other third party material in this article are included in the article's Creative Commons license, unless indicated otherwise in a credit line to the material. If material is not included in the article's Creative Commons license and your intended use is not permitted by statutory regulation or exceeds the permitted use, you will need to obtain permission directly from the copyright holder. To view a copy of this license, visit http://creativecommons.org/ licenses/by/4.0/.

(C) The Author(s) 2017 